FOB-SH: Fragment orbital-based surface hopping for charge carrier transport in organic and biological molecules and materials

J. Spencer, F. Gajdos, and J. Blumberger

Citation: J. Chem. Phys. 145, 064102 (2016); doi: 10.1063/1.4960144

View online: http://dx.doi.org/10.1063/1.4960144

View Table of Contents: http://aip.scitation.org/toc/jcp/145/6

Published by the American Institute of Physics 


\title{
FOB-SH: Fragment orbital-based surface hopping for charge carrier transport in organic and biological molecules and materials
}

\author{
J. Spencer, F. Gajdos, and J. Blumbergera) \\ Department of Physics and Astronomy, University College London, Gower Street, \\ London WC1E 6BT, United Kingdom
}

(Received 19 April 2016; accepted 20 July 2016; published online 9 August 2016)

\begin{abstract}
We introduce a fragment orbital-based fewest switches surface hopping method, FOB-SH, designed to efficiently simulate charge carrier transport in strongly fluctuating condensed phase systems such as organic semiconductors and biomolecules. The charge carrier wavefunction is expanded and the electronic Hamiltonian constructed in a set of singly occupied molecular orbitals of the molecular sites that mediate the charge transfer. Diagonal elements of the electronic Hamiltonian (site energies) are obtained from a force field, whereas the off-diagonal or electronic coupling matrix elements are obtained using our recently developed analytic overlap method. We derive a general expression for the exact forces on the adiabatic ground and excited electronic state surfaces from the nuclear gradients of the charge localized electronic states. Applications to electron hole transfer in a model ethylene dimer and through a chain of ten model ethylenes validate our implementation and demonstrate its computational efficiency. On the larger system, we calculate the qualitative behaviour of charge mobility with change in temperature $T$ for different regimes of the intermolecular electronic coupling. For small couplings, FOB-SH predicts a crossover from a thermally activated regime at low temperatures to a band-like transport regime at higher temperatures. For higher electronic couplings, the thermally activated regime disappears and the mobility decreases according to a power law. This is interpreted by a gradual loss in probability for resonance between the sites as the temperature increases. The polaron hopping model solved for the same system gives a qualitatively different result and underestimates the mobility decay at higher temperatures. Taken together, the FOB-SH methodology introduced here shows promise for a realistic investigation of charge carrier transport in complex organic, aqueous, and biological systems. Published by AIP Publishing. [http://dx.doi.org/10.1063/1.4960144]
\end{abstract}

\section{INTRODUCTION}

The transport of charge carriers through organic and biological semiconductors is at the heart of many exciting technologies such as organic electronics, organic lightemitting diodes and photovoltaics, and nanobioelectronics. While important progress has been made by several groups in recent years towards theory and computational modeling of charge transport (CT) in organic semiconductors (OSs), ${ }^{1-10}$ the nature of excess electrons and electron holes in these materials, as well as their transport mechanism, is still not very well understood. There are two crucial differences with respect to inorganic semiconductors that make the theoretical treatment challenging. First, OS is characterized by a high degree of thermal fluctuations, as the molecules forming the material are held together by the relatively weak van der Waals interactions. Second, the dielectric constant of OS is very low, usually not exceeding 4-6. As we will discuss below, these two materials' characteristics place CT in OS in a difficult regime, where common assertions of standard theories, such as band theory or small polaron hopping, are often not valid. ${ }^{6,710-13}$

The band-like description relies on "Bloch" states and breaks down for ambient temperatures $(T)$ where the mean

\footnotetext{
a)e-mail: j.blumberger@ucl.ac.uk
}

free path is comparable to intermolecular lattice spacings. ${ }^{14,15}$ This theory does not take into account the effects of strong, anharmonic thermal molecular motions on charge transport, which are particularly pronounced in OS due to the weak van der Waals interactions. On the other hand, the thermally activated charge (or small polaron) hopping mechanism, which is often assumed in the literature, is problematic, at least for single crystalline materials without deep traps. See, e.g., Refs. 11-13 and 16 for the case of fullerene single crystals. Activation energies for charge hopping are typically very small in these materials $(0.1 \mathrm{eV})$ and may even disappear for certain molecular configurations, as illustrated in Figure 1. This is due to small reorganization energy $\lambda$ (also termed local electron-phonon coupling), which is a consequence of the low dielectric constant of OS, in combination with relatively large electronic couplings between the molecules, $H_{\mathrm{ab}}$. For configurations where $H_{\mathrm{ab}} \geq \lambda / 2$, the energy barrier for CT disappears (assuming equal site energies) and localized charge carriers no longer form.

What makes CT in OS complex is the strong coupling between electron and nuclear dynamics. Since nuclear thermal fluctuations are large, they may not be treated as a perturbation of the electronic problem, as is done in band theory or Greens function approaches, while the electronic problem may not be integrated out and treated as a perturbation of 


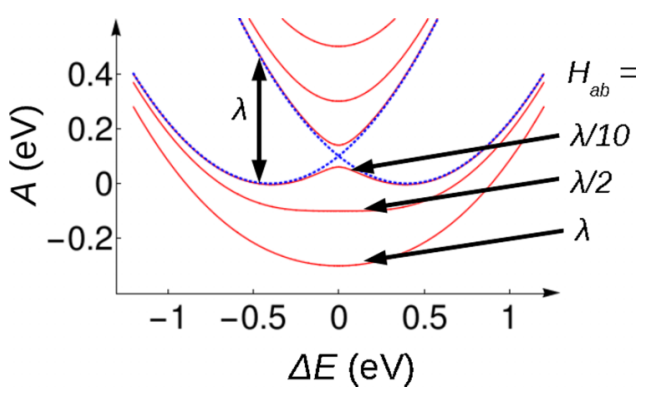

FIG. 1. Adiabatic (red) and diabatic (blue) free energy surfaces for electron transfer. The adiabatic surfaces are plotted for three different values of the electronic coupling matrix element (transfer integral) $H_{\mathrm{ab}}$ for constant reorganization free energy $\lambda . \Delta E$ is the diabatic energy gap (or site energy difference) Eq. (51).

the nuclear problem, as is done in hopping models. What is needed is a unified framework that is able to treat both the electron and nuclear dynamics at an equal footing. Then an unbiased description of the CT mechanism is possible, which can encompass a broad spectrum ranging from delocalized band transport, polaronic hopping, disorder-induced hopping, carrier tunneling, and combinations thereof. Several theoretical frameworks have been developed to treat coupled electron-nuclear dynamics including multi-configurational time-dependent Hartree, ${ }^{17,18}$ ab initio multiple spawning, ${ }^{19,20}$ exact factorization of the molecular wavefunction, ${ }^{21-23}$ and mixed quantum-classical non-adiabatic molecular dynamics (MQC-NAMD $)^{23-25}$ simulation such as Ehrenfest ${ }^{24}$ and fewest switches surface hopping (FSSH) MD. ${ }^{25}$ Here we focus on the latter method as it is well tested and practical for large systems.

Fewest Switches Surface Hopping MD (SH-MD) ${ }^{25}$ is well established in the theoretical chemistry community for simulation of non-adiabatic processes, in particular, ultrafast photo-induced relaxation of molecules and chemical reactions. Several reviews exist that discuss the merits and shortcomings of this method, ${ }^{26-30}$ also in the context of CT in OS. ${ }^{31}$ In SH-MD, the adiabatic electronic states are propagated in time according to the time-dependent electronic Schrödinger equation and the nuclei evolve classically on adiabatic electronic states. The classical nuclear motion creates a time dependent external potential that couples to the electronic degrees of freedom, whereas the quantum back reaction from the electrons to the nuclei is incorporated by stochastic hops of the nuclei between the different adiabatic electronic states. The calculation of the latter is usually the time-limiting factor. A wide spectrum of electronic structure methods has been combined with SH ranging from $a b$ initio wavefunction methods (multireference configuration interaction (MRCI) ${ }^{32} \mathrm{CI},{ }^{33}$ complete active space self-consistent field (CASSCF) ${ }^{32,34,35}$ ), to density functional theory (DFT)-based methods (time-dependent DFT (TDDFT), ${ }^{36-38}$ open-shell Kohn Sham ${ }^{39,40}$ ), to semiempirical approaches such as self-consistent charge density functional tight binding (SCC-DFTB) $)^{41-46}$ and time-dependent SCCDFTB $,{ }^{47,48} \mathrm{OM} 3,{ }^{49,50}$ AM $1,{ }^{51,52}$ and Pariser-Parr-Pople (PPP) electronic Hamiltonians. ${ }^{5,53}$ Whereas the $a b$ initio methods are in practice limited to very small systems, semi-empirical methods can be used to tackle large systems of several hundreds to thousands of atoms. ${ }^{5,42,44-46,52}$

MQC-NAMD approaches targeted specifically for the modeling of CT in extended condensed phase systems and bulk materials have been reported, but they are less well developed than the above mentioned methods for molecular photoexcitation processes. Troisi and co-workers modeled CT in OS by a 1D chain of displaced harmonic oscillators described by an idealized model Hamiltonian, and integrated the time dependent Schrödinger equation for an excess charge moving along this chain. ${ }^{54-56}$ The model could successfully reproduce the temperature dependence of the mobility in rubrene. ${ }^{55}$ Wang and Beljonne used a similar model Hamiltonian approach for a 1D chain of displaced harmonic oscillator within a $\mathrm{SH}$ scheme to propagate the charge carrier. ${ }^{8,57}$ Ren et al. have developed a MQC-NAMD scheme for CT in OS and applied it to a monolayer of a pentathiophene derivative. While their scheme goes beyond harmonic oscillator models and incorporates explicit atomistic detail, the back reaction from the electronic to the nuclear degrees of freedom was omitted ${ }^{7}$ preventing the possibility of spontaneous localization and delocalization of the charge carrier (i.e., polaron formation). Recently, Kubař and Elstner have extended their SCC-DFTBbased MQC-NAMD approach, initially developed for CT in biomolecules, ${ }^{43-45}$ to OS materials and computed hole mobilities in anthracene and a hexabenzocoronene derivative on the level of Ehrenfest MD. ${ }^{10}$

In this contribution, we report on an implementation of SH-MD that is designed to efficiently propagate an excess electron or electron hole in strongly fluctuating structures such as OS materials or large redox active biomolecules. The focus here is on efficiency and on practical applications to real materials comprising several thousands or ten-thousands of atoms. Inspired by electron transfer theory ${ }^{58,59}$ and constrained density functional theory (CDFT),${ }^{60-64}$ we construct the timedependent electronic wavefunction in a charge localized electronic state basis, also termed diabatic basis, although we avoid the term "diabatic" here as the non-adiabatic coupling vectors between the charge localized states are non-vanishing. In principle, each charge localized electronic state may be a correlated open-shell wavefunction obtained from the adiabatic electronic states via a charge localization procedure, or in case of CDFT, a Kohn-Sham determinant with the excess electron or hole constrained on a given molecular site. To make computations practical, however, we follow the notion of an "excess charge carrier," and assert that the complicated many-body or multi-determinantal electron dynamics can be effectively described by a one-particle wavefunction for an excess charge moving in an effective, time-dependent potential due to the other electrons and the nuclei.

In our method, this charge carrier wavefunction is expanded in a basis of fragment (or site) orbitals that mediate the CT, more specifically the singly occupied molecular orbitals (SOMOs) of the molecules forming the material, as obtained from DFT calculations. Hence we term our method "fragment orbital-based SH" (FOB-SH). The dynamics of the remaining valence and core electrons of the molecules is not explicitly treated. The diagonal matrix elements (site energies) of the electronic Hamiltonian in the DFT SOMO basis are 
approximated with a classical force field and the off-diagonal matrix elements (electronic couplings) are calculated using our recently developed analytic overlap method (AOM). ${ }^{65}$ Diagonalization of the electronic Hamiltonian thus obtained gives the adiabatic electronic states used for the SH-MD simulation of CT.

FOB-SH is a minimalistic but atomistic MQC-NAMD method that uses parametrized electronic structure information to propagate the charge carrier wavefunction. In this sense, it shares similarities with the SCC-DFTB-based SH-MD method developed by Kubař and Elstner, but it differs in the way the electronic Hamiltonian matrix elements and nuclear forces are computed. Our method has a number of beneficial features: (i) It is fast because the charge carrier dynamics is propagated in terms of the parametrized DFT SOMOs rather than full charge transfer determinants. The approximation made when replacing determinants by SOMOs for the calculation of off-diagonal matrix elements has been scrutinized before for pi-conjugated systems. ${ }^{65-67} \mathrm{We}$ found this to be a good and useful approximation in most cases, with systematic errors that can be corrected in a straightforward manner. (ii) It should be relatively straightforward to make FOB-SH linear scaling with system size because the electronic Hamiltonian in the SOMO basis is sparse. Therefore, applications to very large condensed phase systems should be feasible. (iii) Working in a charge-localized basis, we minimize the self-interaction error of density functionals that hampers the correct description of adiabatic electronic CT states. Though we note that in-roads have been made recently to address this problem by enforcing the integer-derivative discontinuity. ${ }^{68-70}$

There are some restrictions as well in this first version. (i) We describe CT only within the band formed by the SOMOs (intra-band CT). (ii) We assume that there is only a single charge carrier present as would be the case after injection of an electron or electron hole in a large junk of material (i.e., CT at very low carrier concentration). (iii) Charge separation processes where holes and electrons are present at the same time, as well as superexchange processes where holes and electrons contribute simultaneously to CT via different bridge levels, cannot be currently treated. But our scheme may be suitably extended to describe such situations in future.

In a first application of FOB-SH, we investigate CT in a linear chain of ethylene-like molecules (ELMs). We find that at low electronic coupling strengths between the molecules, the temperature dependence of the charge mobility goes through a maximum, indicative of a crossover from activated transport at low $T$ to band-like transport at high $T$. For sufficiently high electronic coupling strengths, the activated regime disappears and the mobility decreases with increasing $T$ over the entire temperature range. The results are in qualitative agreement with previous $\mathrm{SH}$ simulations using harmonic oscillator model Hamiltonians. ${ }^{8}$

This paper is organized as follows. In Section II A, we give the basic equations for $\mathrm{SH}$, and in Section II B, we define the site basis representation of the charge carrier wavefunction and give details on the construction of the electronic Hamiltonian and non-adiabatic coupling matrix elements (NACEs) in the site basis, and on the transformation of these quantities to the adiabatic representation. In Section II C, we derive an expression for the nuclear forces in the site basis representation and give explicit expressions for their transformation to the adiabatic representation, which is used in FOB-SH. In Section III A, we explain in detail the ultrafast calculation of the electronic Hamiltonian matrix elements, non-adiabatic coupling elements, and non-adiabatic coupling vectors (NACVs) in the site basis using our recently developed analytic overlap method (AOM). After a summary of the simulation details in Sec. IV, we present a validation of the implementation in Sec. V. In particular, we validate the nuclear force calculation on the adiabatic surfaces by comparison to the exact analytic expression for a 2-site model and by monitoring the conservation of total energy during the FOB-SH run for a 10-site model. Then we report on the results of our first application, CT in a chain of ethylene-like molecules. This work is concluded in Sec. VI.

\section{THEORY}

\section{A. Fewest switches surface hopping (FSSH)}

The FSSH algorithm proposed by Tully $^{25}$ has been extensively reviewed. ${ }^{26-30}$ Here we summarize the basic equations that will be referred to in Secs. II B-II D. In the FSSH method, the electronic Schrödinger equation is solved for the time-dependent electronic potential due to classical nuclear motion. The time-dependent electronic wavefunction $\Psi(t)$ is usually expressed as a linear combination of adiabatic electronic wavefunctions $\psi_{n}(\mathbf{R}(t))$ that depend parametrically on the nuclear coordinates $\mathbf{R}(t), \Psi(t)=\sum_{n} c_{n}(t) \psi_{n}(\mathbf{R}(t))$, and $c_{n}$ are the time-dependent expansion coefficients. Insertion in the time-dependent electronic Schrödinger equation gives the time evolution in terms of the expansion coefficients,

$$
i \hbar \dot{c}_{m}(t)=\sum_{n} c_{n}(t)\left(H_{m n}^{\mathrm{ad}}-i \hbar d_{m n}^{\mathrm{ad}}\right),
$$

where $H_{m n}^{\text {ad }}$ are the matrix elements of the electronic Hamiltonian in the adiabatic basis (superscript "ad"), $H_{m n}^{\text {ad }}$ $=\left\langle\psi_{m}|H| \psi_{n}\right\rangle=E_{n} \delta_{m n}$, and $d_{m n}^{\text {ad }}$ are the non-adiabatic coupling matrix elements (NACEs),

$$
d_{m n}^{\mathrm{ad}}=\left\langle\psi_{m} \mid \frac{d \psi_{n}}{d t}\right\rangle .
$$

The classical nuclei evolve on a single adiabatic electronic energy surface at any given time according to Newton's equation of motion. For potential energy surface $n=i, E_{i}$,

$$
m_{I} \ddot{\mathbf{R}}_{I}=-\nabla_{\mathbf{R}_{I}} E_{i} \equiv \mathbf{F}_{I, i},
$$

where $m_{I}$ is the mass and $\mathbf{R}_{I}$ the position vector of nucleus I. Time derivatives are denoted by a dot. The feedback from the electronic to the nuclear subsystem is incorporated in the ability of the system to undergo stochastic "surface hops," i.e., switches from one potential energy surface to another. In the FSSH algorithm devised by Tully, the probability $g_{j i}$ of switching from the current adiabatic electronic state $i$ to another adiabatic electronic state $j$ is calculated from

$$
g_{j i}=\frac{b_{j i} \Delta t}{a_{i i}},
$$




$$
\begin{aligned}
& b_{j i}=-2 \operatorname{Re}\left(a_{j i}^{*} d_{j i}^{\mathrm{ad}}\right), \\
& a_{j i}=c_{j} c_{i}^{*},
\end{aligned}
$$

where $\Delta t$ is the MD time step and $a_{j i}$ the elements of the density matrix.

\section{B. Site basis representation}

In this work, we are interested in describing the evolution of an excess electron or hole through a molecular material. Here we consider the case of an excess electron in a material made of $M$ sites, where "site" refers to a molecule or molecular fragment of the material but note that the formalism is analogous for an excess hole. To this end, the time-dependent electronic wavefunction, $\Psi(t)$, is expanded in a set of timedependent electronic wavefunctions with the excess electron localized at site $l,\left\{\phi_{l}\right\}$,

$$
\Psi(t)=\sum_{l=1}^{M} u_{l}(t) \phi_{l}(\mathbf{R}(t)) .
$$

In principle, the $M$ wavefunctions $\phi_{l}$ can be $N$-electron determinants with the excess electron localized at site $l$ using a suitable localization procedure such as Generalized MullikenHush (GMH) or constrained DFT (CDFT). However, as mentioned in the Introduction, here we restrict ourselves to an effective 1-electron description where the $\left\{\phi_{l}\right\}$ are a set of $M$ orthogonal 1-electron fragment (or site) orbitals, rather than $N$-electron wavefunctions. The site orbitals are obtained from the singly occupied molecular orbitals (SOMOs) of the isolated molecules, in the following denoted $\left\{\varphi_{m}\right\}$, via Löwdin orthogonalization:

$$
\phi_{l}=\sum_{m=1}^{M} T_{m l} \varphi_{m}
$$

where $T_{m l}$ are the elements of the matrix $\mathbb{T}, T_{m l} \equiv[\mathbb{T}]_{m l}$ $=\left[\mathbb{S}^{-1 / 2}\right]_{m l}$ and $\mathbb{S}$ the overlap matrix with elements $S_{m l}$ $\equiv[\mathbb{S}]_{m l}=\left\langle\varphi_{m} \mid \varphi_{l}\right\rangle$. The SOMOs $\left\{\varphi_{m}\right\}$ can be obtained from gas phase calculations on the isolated molecules in vacuum for the same atomic configuration as in the material and for the charge state $1-\left(1+\right.$ for holes). Hence, while the $\left\{\varphi_{m}\right\}$ are strictly localized on site $m$ and have zero amplitude anywhere else, the $\left\{\phi_{m}\right\}$ are strongly localized on site $m$ and have small tails on the neighboring sites to enforce orthogonality. As an illustrative example, we show in Figure $2\left\{\varphi_{m}\right\}$ for a dimer and a chain of ethylene-like molecules, in this case the $\pi_{2 \mathrm{p}}$ molecular orbital of each molecule.

Insertion of Eq. (7) in the time-dependent electronic Schrödinger equation and multiplication from the left with $\phi_{k}$ and integration give

$$
i \hbar \dot{u}_{k}(t)=\sum_{l} u_{l}(t)\left(H_{k l}-i \hbar d_{k l}\right),
$$

where $H_{k l}$ are the matrix elements of the electronic Hamiltonian $H$ and $d_{k l}$ the non-adiabatic coupling elements (NACEs) in the orthogonal site orbital basis, respectively,

$$
\begin{aligned}
H_{k l} & \equiv[\mathbb{H}]_{k l}=\left\langle\phi_{k}|H| \phi_{l}\right\rangle, \\
d_{k l} & \equiv[\mathbb{D}]_{k l}=\left\langle\phi_{k} \mid \dot{\phi}_{l}\right\rangle .
\end{aligned}
$$
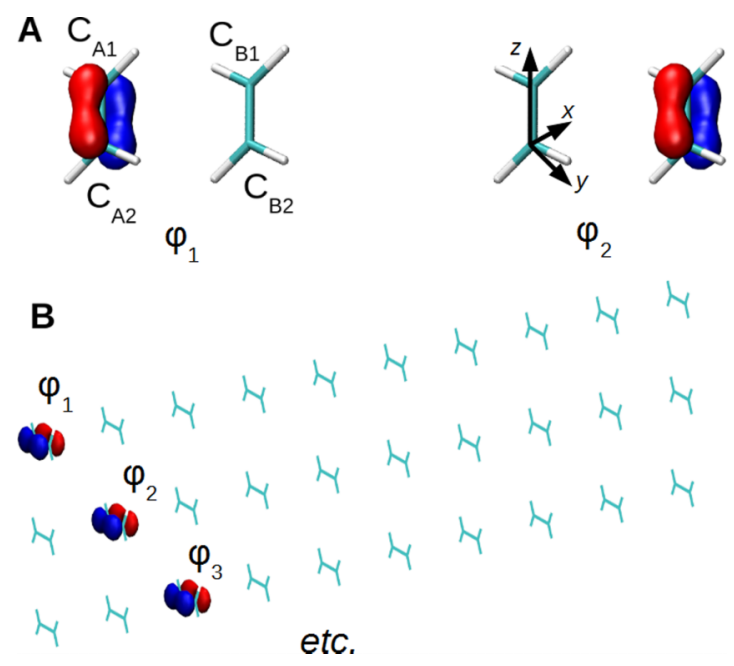

FIG. 2. Systems simulated in this work using FOB-SH. (a) Electron hole transfer in a ethylene-like molecule (ELM) donor-acceptor complex and (b) electron transport in a 1D chain of 10 ELMs. In (b) the chain is replicated to illustrate the SOMOs of the positively charged ELMs $\left(\varphi_{1}, \varphi_{2}, \varphi_{3}, \ldots\right)$ in blue and red isosurfaces. They are used as basis functions for expansion of the hole carrier wavefunction $\Psi(t)$, Eqs. (7) and (8). Carbon atoms are drawn in green. The directions $x$ and $z$ are labelled for comparison with Table I: $x$ is the direction pointing along the direction of stacking, while $z$ points along the long axis of the molecule.

Eq. (9) determines the time evolution of the excess electron in terms of the expansion coefficients in the orthogonal site orbital basis $\left\{\phi_{l}\right\}$. The matrix elements $H_{k l}$ and $d_{k l}$ can be expressed in terms of the non-orthogonal site orbital basis $\left\{\varphi_{l}\right\}$ by substitution of Eq. (8),

$$
\begin{aligned}
H_{k l} & =\left[\mathbb{T}^{\dagger} \mathbb{V}^{\prime} \mathbb{T}\right]_{k l}=\left[\mathbb{T}^{-1} \mathbb{H} \mathbb{H}^{\prime} \mathbb{T}\right]_{k l}, \\
d_{k l} & =\left[\mathbb{T}^{\dagger} \mathbb{D}^{\prime} \mathbb{T}\right]_{k l}+\left[\mathbb{T}^{\dagger} \mathbf{S}\right]_{k l},
\end{aligned}
$$

where

$$
\begin{aligned}
V_{k l}^{\prime} & \equiv\left[\mathbb{V}^{\prime}\right]_{k l}=\left\langle\varphi_{k}|H| \varphi_{l}\right\rangle, \\
d_{k l}^{\prime} & \equiv\left[\mathbb{D}^{\prime}\right]_{k l}=\left\langle\varphi_{k} \mid \dot{\varphi}_{l}\right\rangle,
\end{aligned}
$$

and

$$
\mathbb{H}^{\prime}=\mathbb{S}^{-1} \mathbb{V}^{\prime}
$$

is the electronic Hamiltonian in the non-orthogonal site orbital basis. As we will see in Section III, the Hamiltonian will be constructed in the orthogonal basis, which requires us to solve the standard eigenvalue equation for $\mathbb{H}$ rather than the generalized eigenvalue equation for $\mathbb{V}^{\prime}$.

During the surface hopping simulation, the nuclei are propagated on one of the adiabatic electronic potential energy surfaces according to Eq. (3), and the probability for hopping from adiabatic electronic state $i$ to another state $j$, Eq. (4), needs to be calculated at every MD time step. This requires the knowledge of the adiabatic electronic energies $E_{n}$ and the NACEs between the adiabatic electronic states $\psi_{n}$. They can be easily obtained from the site orbital representation via a unitary transformation,

$$
\psi_{n}(t)=\sum_{l} U_{l n} \phi_{l}(t)
$$


where $U_{l n} \equiv[\mathbb{U}]_{l n}$ and $\mathbb{U}$ diagonalizes $\mathbb{H}$,

$$
\mathbb{H}^{\text {ad }}=\mathbb{U}^{\dagger} \mathbb{H} \mathbb{U},
$$

with $E_{n}=\left[\mathbb{H}^{\mathrm{ad}}\right]_{n n}$.

For calculation of the NACEs, we insert the unitary transformation Eq. (17) in Eq. (2). This gives for $n=j, i$,

$$
d_{j i}^{\mathrm{ad}}=\left[\mathbb{U}^{\dagger} \mathbb{D} \mathbb{U}\right]_{j i}+\left[\mathbb{U}^{\dagger} \mathbb{U}_{j i},\right.
$$

where $\mathbb{D}$ is the matrix of NACEs in the site orbital basis, Eq. (11). In Section II C, we discuss how the nuclear gradients on the adiabatic electronic states and hence the forces for MD simulation can be obtained from the gradients in the site basis representation.

\section{Nuclear gradients}

The force on nucleus $I$ on the adiabatic potential energy surface $E_{i}$ is

$$
\mathbf{F}_{I, i}=-\nabla_{I}\left\langle\psi_{i}|H| \psi_{i}\right\rangle=-\left\langle\psi_{i}\left|\nabla_{I} H\right| \psi_{i}\right\rangle,
$$

where we have used the Hellmann-Feynman theorem in the last equation. Unfortunately, the expectation value of the gradient of the Hamiltonian is not available in our approach as we do not construct explicit expressions of the adiabatic wavefunctions $\psi_{i}$ in terms of spatial coordinates. However, the nuclear force can be rigorously computed from the gradient of the Hamiltonian matrix elements in the site basis $\left\{\phi_{l}\right\}$, which are readily available in our scheme,

$$
\mathbf{F}_{I, i}=-\left[\mathbb{U}^{\dagger}\left(\nabla_{I} \mathbb{H}\right) \mathbb{U}\right]_{i i}
$$

where

$$
\left[\nabla_{I} \mathbb{H}\right]_{k l} \equiv \nabla_{I} H_{k l}=\nabla_{I}\left\langle\phi_{k}|H| \phi_{l}\right\rangle .
$$

A similar expression has been used before in the context of CDFT configuration interaction. ${ }^{71}$ Equation (21) implies that nuclear derivatives of the transformation matrix $\mathbb{U}$ are not needed. To show this we insert Eq. (17) for $n=i$ in the RHS of Eq. (20) to obtain

$$
\mathbf{F}_{I, i}=-\left[\mathbb{U}^{\dagger} \mathbb{G}_{I} \mathbb{U}\right]_{i i},
$$

with matrix elements $\left[\mathbb{G}_{I}\right]_{k l}=\left\langle\phi_{k}\left|\nabla_{I} H\right| \phi_{l}\right\rangle$. The latter elements can be written as

$$
\begin{aligned}
\left\langle\phi_{k}\left|\nabla_{I} H\right| \phi_{l}\right\rangle & =\nabla_{I}\left\langle\phi_{k}|H| \phi_{l}\right\rangle-\left\langle\nabla_{I} \phi_{k}|H| \phi_{l}\right\rangle-\left\langle\phi_{k}|H| \nabla_{I} \phi_{l}\right\rangle \\
& =\nabla_{I} H_{k l}-\left\langle\nabla_{I} \phi_{k} \mid \sum_{m} H_{m l} \phi_{m}\right\rangle-\left\langle\nabla_{I} \phi_{l} \mid \sum_{m} H_{m k} \phi_{m}\right\rangle^{*} \\
& =\nabla_{I} H_{k l}+\sum_{m}\left(\mathbf{d}_{I, k m} H_{m l}-H_{k m} \mathbf{d}_{I, m l}\right),
\end{aligned}
$$

where

$$
\mathbf{d}_{I, k l} \equiv\left[\mathbb{D}_{I}\right]_{k l}=\left\langle\phi_{k} \mid \nabla_{I} \phi_{l}\right\rangle
$$

are the NACVs in the orthogonal site basis. In Eq. (26), we have made use of the identity

$$
\mathbf{d}_{I, k m}=-\mathbf{d}_{I, m k}^{*}
$$

which is valid for orthogonal wavefunctions, and the star on the right hand side of Eq. (28) denotes the complex conjugate. Thus, in matrix notation, $\mathbb{G}_{I}$ in Eq. (23) is given by

$$
\mathbb{G}_{I}=\nabla_{I} \mathbb{H}+\left[\mathbb{D}_{I}, \mathbb{H}\right]
$$

where the second term on the RHS of Eq. (29) is the commutator $\left[\mathbb{D}_{I}, \mathbb{H}\right]=\mathbb{D}_{I} \mathbb{H}-\mathbb{H} \mathbb{D}_{I}$, and $\left[\mathbb{D}_{I}, \mathbb{H}\right] \neq 0$ in general. To proceed, we note that we are only interested in the diagonal elements of the matrix on the RHS of Eq. (23). It can be easily shown that the diagonal elements of the transformed commutator vanish,

$$
\begin{aligned}
{\left[\mathbb{U}^{\dagger}\left[\mathbb{D}_{I}, \mathbb{H}\right] \mathbb{U}\right]_{i i} } & =\left[\mathbb{U}^{\dagger} \mathbb{D}_{I} \mathbb{H} \mathbb{U}-\mathbb{U}^{\dagger} \mathbb{H} \mathbb{D _ { I }} \mathbb{U}_{i i}\right. \\
& =\left[\mathbb{U}^{\dagger} \mathbb{D}_{I} \mathbb{U} \mathbb{U}^{\dagger} \mathbb{H} \mathbb{U}\right]_{i i}-\left[\mathbb{U}^{\dagger} \mathbb{H} \mathbb{U} \mathbb{U}^{\dagger} \mathbb{D}_{I} \mathbb{U}\right]_{i i} \\
& =\left[\mathbb{U}^{\dagger} \mathbb{D}_{I} \mathbb{U}\right]_{i i} E_{i}-E_{i}\left[\mathbb{U}^{\dagger} \mathbb{D}_{I} \mathbb{U}\right]_{i i} \\
& =0 .
\end{aligned}
$$

Hence, inserting Eqs. (29) and (33) in Eq. (23) gives Eq. (21).

\section{Special case: Donor-acceptor complex}

In the special case of ET in a donor-acceptor complex (2 sites only), the transformation from the orthogonal site basis to the adiabatic basis is analytic. For a $2 \times 2$ matrix, Eq. (18) gives the usual expression

$E_{0 / 1}(\mathbf{R})=\frac{H_{11}(\mathbf{R})+H_{22}(\mathbf{R})}{2} \pm \frac{1}{2} \sqrt{\Delta E^{2}(\mathbf{R})+4 H_{12}^{2}(\mathbf{R})}$,

where on the RHS of Eq. (34), the minus sign is for the adiabatic ground state energy, $E_{0}$, and the plus sign for the excited adiabatic state, $E_{1}$, and $\Delta E$ is the vertical site (diabatic) energy gap,

$$
\Delta E(\mathbf{R})=H_{22}(\mathbf{R})-H_{11}(\mathbf{R}) .
$$

Hence, the forces on the two adiabatic surfaces can be directly obtained by differentiation of Eq. (34). The more tedious route in Section II C for the general $N$-site case can be avoided. The resultant expression

$$
\begin{aligned}
\mathbf{F}_{I, 0 / 1}= & -\frac{\nabla_{I} H_{11}+\nabla_{I} H_{22}}{2} \\
& \mp \frac{\Delta E\left(\nabla_{I} H_{22}-\nabla_{I} H_{11}\right)+4 H_{12} \nabla_{I} H_{12}}{2 \sqrt{\Delta E^{2}+4 H_{12}^{2}}}
\end{aligned}
$$

is, of course, equivalent to Eq. (21) $(i=0,1)$. 


\section{IMPLEMENTATION}

The formulation of surface hopping in the site basis in Sections II B and II C requires the calculation of a number of matrix elements and nuclear derivatives. For propagation of the electronic subsystem according to Eq. (9), one needs to calculate the electronic Hamiltonian matrix elements $H_{k k}$, $H_{k l}$, as well as the non-adiabatic coupling elements (NACEs) $d_{k l}$. For propagation of the nuclear subsystem with forces given by Eq. (21), one needs to calculate at each MD time step the nuclear gradients $\nabla_{I} H_{k k}, \nabla_{I} H_{k l}$, and the unitary transformation matrix $U_{k l}$. In the following, we describe computationally efficient strategies for the calculation of these quantities.

\section{A. Diagonal matrix elements (site energies) $\boldsymbol{H}_{k k}$}

The site orbital basis functions $\left\{\phi_{k}\right\}$ are strongly localized on site $k$ and typically have only a very small contribution on neighboring sites $l$ to enforce orthogonality. The corresponding site energies $H_{k k}=\left\langle\phi_{k}|H| \phi_{k}\right\rangle$ are approximated here at the classical force field level. The site $k$ carries the total excess electron whereas all other sites $l \neq k$ are modeled in their neutral charge state. The force field parameters for charged and neutral states of the sites may be taken from standard force fields or obtained from electronic structure calculations of the isolated molecules. The total site energy is then computed for the full condensed phase system with site $k$ in the charged state and sites $l \neq k$ in the neutral state applying periodic boundary conditions. Condensed phase electronic polarization effects can be accounted for using, e.g., electronically polarizable force fields.

\section{B. Off-diagonal matrix elements (electronic couplings) $\boldsymbol{H}_{k l}$}

The off-diagonal elements $H_{k l}, l \neq k$, are an intrinsically quantum mechanical property. For their calculation, we consider at first the non-orthogonal site orbitals $\left\{\varphi_{k}\right\}$ and the corresponding overlap matrix elements $S_{k l}$. As mentioned before, we restrict ourselves to a 1-electron description and identify $\varphi_{k}$ simply with the SOMO of molecule $k$, and similarly for molecule $l$. The SOMO is obtained from standard electronic structure calculation on the isolated molecule in the charged state. The SOMO is then represented in a minimum basis of Slater-type atomic orbitals (STOs) with optimized Slater decay coefficients. For $\pi$-conjugated systems, it is usually sufficient to include only one optimized Slater $p$-orbital per atom contributing to $\pi$-conjugation. In this case

$$
S_{k l}=\left\langle\varphi_{k} \mid \varphi_{l}\right\rangle=\sum_{i \in k}^{\text {atoms atoms }} \sum_{j \in l}^{*} c_{p \pi, i}^{*} c_{p \pi, j}\left\langle p_{\pi, i} \mid p_{\pi, j}\right\rangle,
$$

where $i$ and $j$ run over all $\pi$-conjugated atoms in molecules $k$ and $l$, respectively, $p_{\pi, i}$ is the STO $p$ orbital on atom $i$ pointing along the direction of pi-conjugation and $c_{p \pi, i}$ is the corresponding expansion coefficients. The latter is obtained from projection of $p_{\pi, i}$ on the SOMO obtained from explicit electronic structure calculation with a standard basis set. (In our previous work, we have denoted the double sum on the right hand side of Eq. (37) by $\bar{S}_{k l}{ }^{65}$ ) The overlap matrix elements of Eq. (37) are used to obtain $H_{k l}$ according to the linear relationship

$$
H_{k l}=C S_{k l},
$$

where $C$ is a constant of proportion. Equation (38) asserts that the off-diagonal Hamiltonian matrix element between the orthogonal site orbitals $\left(\phi_{k} \phi_{l}\right), H_{k l}$, is proportional to the orbital overlap of the corresponding non-orthogonal site orbitals $\left(\varphi_{k}, \varphi_{l}\right), S_{k l}$, where the two orbital pairs are related by the Lowdin transformation Eq. (8). The estimation of $H_{k l}$ according to Eq. (38) with overlaps computed according to Eq. (37) is denoted as analytic overlap method (AOM). The crucial advantage of this scheme is that the calculation of the overlap Eq. (37) and electronic coupling via Eq. (38) is analytic and ultrafast owing to the STO representation of the SOMO.

Equation (38) was suggested a long time ago by LonguetHiggins and Roberts ${ }^{72}$ and seems like a major approximation at first sight. However, we have previously given theoretical arguments for the validity of Eq. (38) in the small overlap regime using the exact expression for $H_{k l}$ within constrained density functional theory. ${ }^{73}$ We have also previously assessed the accuracy of Eq. (38) on a diverse set of $\pi$-conjugated donor-acceptor pairs and found a good linear correlation between $H_{k l}$ and $S_{k l}$ as well as transferability of the relation to other $\pi$-conjugated compounds. ${ }^{65}$ In that study we devised a training set of $\pi$-conjugated organic donor-acceptor pairs (HAB11 database ${ }^{66}$ and $\mathrm{C}_{60}$ ) for which we calculated electronic coupling matrix elements (transfer integrals) $H_{k l}$ at the level of fragment-orbital density functional theory (FODFT $^{12,64,74}$ ), as well as the SOMO overlaps $S_{k l}$ according to Eq. (37). A linear fit $\left(R^{2}=0.974\right)$ gave $C=1.819 \mathrm{eV}$. We found that the same linear relation holds well for a diverse set of $\pi$-conjugated organic donor-acceptor pairs (acenes, thiophene, porphin, imidazole, $\mathrm{C}_{60}$ in random orientations) that were not included in the linear fit (denoted "test set" in Ref. 65). The average error in $H_{k l}$ values obtained from the STO overlap according to Eq. (38) was only a factor of 1.9 , translating into an error in the non-adiabatic ET rate by a factor of 3.6. ${ }^{65}$ Remarkably, this is less than an order of magnitude error in the reaction rate, which is often referred to as "chemical accuracy." Other groups have reported similarly good correlations. Friesner and co-workers noticed a good correlation between overlap and coupling for ET between bacteriochlorophyll cofactors, except for one cofactor pair where deviations were somewhat larger but still reasonable, ${ }^{75}$ and Troisi used Eq. (38) for the estimation of electronic couplings between DNA base pairs. ${ }^{76}$

Although Eq. (38) gives a useful approximation for a wide class of $\pi$-conjugated compounds, it needs to be tested against explicit electronic structure calculations for molecules outside our training and test sets. Other expressions relating overlaps with electronic couplings have been suggested as well, ${ }^{72}$ and they may be used alternatively. Finally, we note that there is a subtle difference between the donor-acceptor pairs investigated in Ref. 65 and the multi-site systems investigated in this work. In the latter, the $\phi_{k}$ have small tails on all neighboring sites $l$, not only on a single site as in case of donor-acceptor compounds. The influence of these additional tails on $H_{k l}$ can be expected to be small and is neglected. 


\section{Time evolution of $\boldsymbol{H}_{k k}, \boldsymbol{H}_{k l}$, and SOMOs}

The diagonal and off-diagonal Hamiltonian matrix elements as well as the SOMOs are time-dependent due to the dynamics of the nuclear subsystem $\mathbf{R}=\mathbf{R}(t), H_{k k}$ $=H_{k k}(\mathbf{R}(t)), H_{k l}=H_{k l}(\mathbf{R}(t))$, and $\varphi_{k}=\varphi_{k}(\mathbf{R}(t))$, see Eq. (9). The time-evolution of the site energies, $H_{k k}(t)$, is simply given by the force field energy of state $k$ along the SH MD trajectory $\mathbf{R}(t)$. The off-diagonal element $H_{k l}(t)$ is proportional to the time dependent overlap $S_{k l}(t)$ between the SOMOs. The question is how to update the SOMO $\varphi_{k}(t)$ efficiently during the SH MD run. In our scheme, the projection coefficients $c_{p \pi, i}$ in Eq. (37) are calculated for the minimum energy structure of molecule $k$ in vacuum and used as an input for the FOB-SH simulation. After every nuclear dynamics time step, the orientation of the atomic orbital $p_{\pi, i}$ is updated so that it remains parallel to the direction of $\pi$-conjugation. This direction is orthogonal to the plane that minimizes the sum of the distances between the plane and atom $i$ and the atoms covalently connected to $i$. The expansion coefficients of all atomic orbitals on molecule $k$ are then scaled by the same factor (typically a small number close to 1 ) to normalize the SOMO at the new geometry. The expansion coefficients are not changed otherwise. Hence, the atomic orbitals comprising the SOMO follow the motion of the atoms but they are frozen otherwise.

One can expect this to be a reasonable approximation if the SOMO is stable against intramolecular vibrations. As we will see in Section V A 1, this is the case for hole transfer in our model ethylene systems. There are, however, situations where this approximation is likely to break down. For instance, in case of degeneracy of the SOMO, certain vibronic modes or environmental effects may lift the degeneracy leading to rapid change of expansion coefficients and strong orbital relaxation. To describe such effects, more elaborate methods are needed for the update of the SOMO along the SH MD trajectory.

\section{Non-adiabatic coupling elements (NACEs)}

Equation (37) is also used for rapid estimation of the NACEs. The time derivative of the site basis function in Eq. (15) is replaced by a finite difference approximation,

$$
\begin{aligned}
d_{k l}^{\prime}(t) & =\left\langle\varphi_{k}(t) \mid \frac{\mathrm{d}}{\mathrm{d} t} \varphi_{l}(t)\right\rangle \\
& \approx \frac{1}{\Delta t}\left(\left\langle\varphi_{k}(t) \mid \varphi_{l}(t+\Delta t)\right\rangle-\left\langle\varphi_{k}(t) \mid \varphi_{l}(t)\right\rangle\right) \\
& =\frac{1}{\Delta t}\left(S_{k l^{\prime}}(t)-S_{k l}(t)\right),
\end{aligned}
$$

where $\Delta t$ is the MD time step, and $S_{k l^{\prime}}$ the overlap between $\varphi_{k}$ at time $t$ and $\varphi_{l}$ at time $t+\Delta t$. Both $S_{k l^{\prime}}$ and $S_{k l}$ are calculated according to Eq. (37). The NACEs in the non-orthogonal site basis, $d_{k l}^{\prime}$, are then transformed to the NACEs in the orthogonal basis, $d_{k l}$, according to Eq. (13). The transformation matrix $T_{k l}$ is simply obtained from $S_{k l}$ via matrix inversion and the time derivative $\dot{T}_{k l}$ is approximated by a finite difference calculation.

\section{E. Nuclear gradients}

For the calculation of the total force Eq. (21), the diagonal contribution of the Hamiltonian, $\nabla_{I} H_{k k}$, is taken from the force field, consistently with our choice for the diagonal energies. The off-diagonal contributions, $k \neq l$, are obtained from Eq. (38),

$$
\nabla_{I} H_{k l}=C \nabla_{I} S_{k l} .
$$

The nuclear gradients of the overlap, $\nabla_{I} S_{k l}$, can be conveniently expressed in terms of the NACVs,

$$
\begin{aligned}
\nabla_{I} S_{k l} & =\nabla_{I}\left\langle\varphi_{k} \mid \varphi_{l}\right\rangle=\left\langle\nabla_{I} \varphi_{k} \mid \varphi_{l}\right\rangle+\left\langle\varphi_{k} \mid \nabla_{I} \varphi_{l}\right\rangle \\
& =\mathbf{d}_{I, l k}^{\prime *}+\mathbf{d}_{I, k l}^{\prime}
\end{aligned}
$$

which are calculated numerically using the finite difference approximation,

$$
\begin{aligned}
\mathbf{d}_{I, k l}^{\prime}= & \left\langle\varphi_{k} \mid \nabla_{I} \varphi_{l}\left(\mathbf{R}_{1}, \ldots, \mathbf{R}_{I}, \ldots, \mathbf{R}_{M}\right)\right\rangle \\
& \approx\left[\begin{array}{l}
\frac{1}{\Delta s}\left(\left\langle\varphi_{k} \mid \varphi_{l}\left(\mathbf{R}_{1}, \ldots, \mathbf{R}_{I}+\Delta s \mathbf{e}_{x}, \ldots, \mathbf{R}_{M}\right)\right\rangle-\left\langle\varphi_{k} \mid \varphi_{l}\left(\mathbf{R}_{1}, \ldots, \mathbf{R}_{I}, \ldots, \mathbf{R}_{M}\right)\right\rangle\right) \\
\frac{1}{\Delta s}\left(\left\langle\varphi_{k} \mid \varphi_{l}\left(\mathbf{R}_{1}, \ldots, \mathbf{R}_{I}+\Delta s \mathbf{e}_{y}, \ldots, \mathbf{R}_{M}\right)\right\rangle-\left\langle\varphi_{k} \mid \varphi_{l}\left(\mathbf{R}_{1}, \ldots, \mathbf{R}_{I}, \ldots, \mathbf{R}_{M}\right)\right\rangle\right) \\
\frac{1}{\Delta s}\left(\left\langle\varphi_{k} \mid \varphi_{l}\left(\mathbf{R}_{1}, \ldots, \mathbf{R}_{I}+\Delta s \mathbf{e}_{z}, \ldots, \mathbf{R}_{M}\right)\right\rangle-\left\langle\varphi_{k} \mid \varphi_{l}\left(\mathbf{R}_{1}, \ldots, \mathbf{R}_{I}, \ldots, \mathbf{R}_{M}\right)\right\rangle\right)
\end{array}\right] \\
& =\left\{\begin{array}{cl}
\frac{1}{\Delta s}\left(\mathbf{S}_{I, k l^{\prime}}-S_{k l} \mathbf{1}\right) & \text { if } I \in l \\
\mathbf{0} & \text { if } I \notin l
\end{array}\right.
\end{aligned}
$$

In Eqs. (43) and (44), $\Delta s$ is a small displacement and $\mathbf{e}_{x}$, $\mathbf{e}_{y}, \mathbf{e}_{z}$ are the unit vectors in $x, y$, and $z$ directions, $\mathbf{S}_{I, k l^{\prime}}$ is the vector of overlaps between $\varphi_{k}$ at nuclear coordinates $\mathbf{R}=\left(\mathbf{R}_{1}, \ldots, \mathbf{R}_{I}, \ldots, \mathbf{R}_{M}\right)$ and $\varphi_{l}$ at nuclear coordinates of atom $I$ displaced by $\Delta s \mathbf{e}_{x}, \Delta s \mathbf{e}_{y}$, and $\Delta s \mathbf{e}_{z}$, respectively (first vector in Eq. (43)), and $\mathbf{0}$ and $\mathbf{1}$ are the null and unity vectors, respectively. Eq. (44) assumes that a displacement of a given atom $I$ on a molecule $l$ only leads to a change in the SOMO for that molecule, i.e., $\varphi_{l}$. The SOMOs of all other sites including site $k$ remain unchanged. This is a consequence of the neglect 
of electronic relaxation effects due to nuclear motion in our scheme, as discussed above.

\section{F. Integration of equations of motion}

The coupled equations of motion for the excess charge carrier, Eq. (9), and classical nuclei, Eq. (3), are integrated numerically using an electronic time step $\delta t$ and a nuclear time step $\Delta t$ for propagation of charge carrier and nuclei, respectively. At the initial time $t$, the electronic Hamiltonian matrix elements $H_{k k}(t)$ and $H_{k l}(t)$, the nuclear gradients $\nabla_{I} H_{k k}$ and $\nabla_{I} H_{k l}$, and the NACEs $d_{k l}^{\prime}(t)$ are calculated as described in Secs. III A-III E. They are transformed to the adiabatic basis according to Eqs. (18), (21), and (13) to obtain the adiabatic energies $E_{i}(t)$, nuclear forces $\mathbf{F}_{I, i}(t)$, and NACEs $d_{k l}(t)$. The nuclei are then propagated on a given adiabatic surface $i$ from time $t$ to $t+\Delta t$ according to Eq. (3) using the velocity Verlet algorithm. This is followed by the calculation of the electronic Hamiltonian matrix elements at time $t+\Delta t$, $H_{k k}(t+\Delta t)$, and $H_{k l}(t+\Delta t)$. In the next step, the expansion coefficients of the charge carrier wavefunction in the site basis, $u_{k}$, are propagated according to Eq. (9) from time $t$ to $t+\Delta t$ in steps of $\delta t$ using the fourth-order Runge-Kutta algorithm. ${ }^{77}$ At each electronic integration time step $n$, the electronic Hamiltonian matrix elements are linearly interpolated between $H_{k l}(t)$ and $H_{k l}(t+\Delta t), H_{k l}(t+n \delta t)=H_{k l}(t)+\left[H_{k l}(t+\Delta t)\right.$ $\left.-H_{k l}(t)\right](n \delta t / \Delta t), n=1, \ldots,(\Delta t / \delta t)$, and similarly for the diagonal elements. The coefficients $u_{k}(t+\Delta t)$ are then transformed to the adiabatic basis $c_{i}(t+\Delta t)$. The latter are used for the calculation of the surface hopping probabilities between the current adiabatic electronic state $i$ and all other states $j \neq i$ according to Eq. (4). When a surface hop from $i$ to $j$ is attempted, the atomic velocities of the system are rescaled by a factor $\kappa=\sqrt{\frac{T_{i}-\left(E_{j}-E_{i}\right)}{T_{i}}}$, where $T_{i}$ is the total kinetic energy of the system in state $i$ and $E_{i}, E_{j}$ are the potential energies in states $i$ and $j$, respectively. If there is not enough kinetic energy to compensate for the difference in potential energy, the hop does not happen. If the hop does happen, the nuclear forces are updated. We note that our velocity rescaling approach differs from the usual approach, where the velocity component in the direction of the non-adiabatic coupling vector is rescaled. Unfortunately, the current implementation of our methodology did not allow us to scale the velocity in that way. As the purpose of this work is to introduce and demonstrate the feasibility of a new methodology rather than report quantitative results, we disregard in this first version of our implementation any possible systematic errors due to the modified velocity rescaling approach.

\section{G. Decoherence correction}

A well-known shortcoming of the $\mathrm{SH}$ method is electronic over-coherence. After passing the crossing region, the off-diagonal electronic density matrix element does not decay sufficiently leading to an overly coherent electronic wavefunction with probability density on two or more surfaces. Several correction schemes have been suggested to mitigate this problem. ${ }^{28,78-85}$ One of the simplest approaches, which we use in the current implementation, is to collapse the charge carrier wavefunction $\Psi(t)$ to the active adiabatic state when the system is sufficiently far from a crossing point. ${ }^{79}$ Application of this procedure to the two model systems that we simulate in this work is explained in more detail in Section IV C.

\section{H. Work flow of FOB-SH simulation}

A simplified work flow is shown in Figure 3. It consists of a preparatory fragment orbital (FO)-parametrization and the actual FOB-SH MD simulation. The FO-parametrization involves three steps. First, standard Kohn-Sham DFT calculations are carried out to obtain the SOMO $\varphi_{k}$ of the molecules or molecular sites that mediate charge transport. Second, the SOMOs are expanded in a minimum basis of Slater-type orbitals with optimized exponential decay coefficients $\bar{\mu}_{2 \mathrm{p}}$ and expansion coefficients $c_{\mathrm{p} \pi, i}$. Third, force field parameters for the neutral and charged states of the molecules need to be chosen either from existing force fields or by parametrization to electronic structure calculations. It is desirable for the force field to reproduce properties that are important for charge transport, in particular, reorganization energy $\lambda$ (Eq. (45)) and ionization potential/electron affinity.

The SOMO parameters $\bar{\mu}_{2 \mathrm{p}}$ and $c_{\mathrm{p} \pi, i}$ as well as the force field parameters are used as an input for the FOB-SH simulation. The FOB-SH program consists of fortran routines for charge carrier propagation which are interfaced by a tcl script with the classical MD package NAMD ${ }^{86}$ (name of the

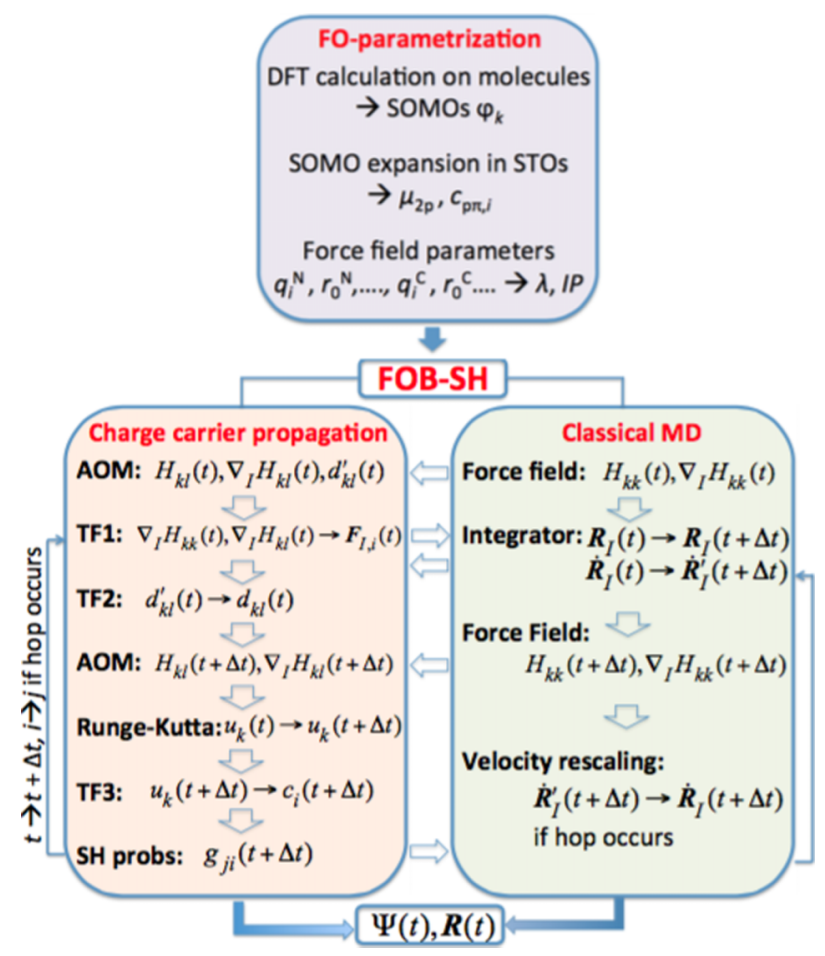

FIG. 3. Simplified workflow of a fragment orbital-based surface hopping (FOB-SH) simulation. FO: fragment orbital, DFT: density functional theory, MD: molecular dynamics, SOMO: singly occupied molecular orbital, AOM: analytic overlap method, TF1-TF3: transformations, SH probs: surface hopping probabilities. See Section III H for an explanation of the scheme. 
package not to be confused with non-adiabatic molecular dynamics). The charge carrier propagation routines read the SOMO parameters and calculate the off-diagonal Hamiltonian matrix elements and nuclear gradients, the NACEs and NACVs as explained in Sections III B-III E. Transformations to the adiabatic electronic states are carried out and the charge carrier wavefunction is propagated in time and surface hopping probabilities are calculated as described in Sections III F and III G. The classical MD package reads the force field parameters for neutral and charged molecules, computes the diagonal Hamiltonian matrix elements and gradients as outlined in Section III A, and propagates the nuclei on the adiabatic electronic states using forces imported from the charge propagation routine. The output of the FOB-SH MD simulation is the time-dependent wavefunction of the charge carrier, $\Psi(t)$, and the nuclear trajectory $\mathbf{R}(t)$.

\section{SIMULATION DETAILS}

\section{A. Model systems}

In a first demonstration and validation of the FOB-SH methodology, we have chosen to simulate electron hole transfer between ethylene-like molecules (ELMs). We call them "ethylene-like" because only their nuclear geometries correspond to real ethylene molecules. The reorganization energy $\lambda$ and the constant $C$ determining the magnitude of $H_{k l}$ (Eq. (38)) are chosen freely to simulate hole transfer in different parameter regimes, see Section IV B for details. The two model systems simulated are shown in Figure 2. Panel A displays a simple donor-acceptor complex, consisting of two ELMs at an average centre-to-centre distance of $4 \AA$. The SOMOs of the left and right ELM, $\varphi_{1}$ and $\varphi_{2}$, respectively, are indicated by isosurfaces. They are used as basis functions for the expansion of the charge carrier wavefunction according to Eqs. (7) and (8) and for the construction of the electronic Hamiltonian. Panel B shows ten ELMs, designed to demonstrate the transferability of the method onto organic molecular systems. Again these molecules are spaced $4 \AA$ apart. Each molecule contributes a SOMO for the expansion of the charge carrier wavefunction and construction of the electronic Hamiltonian. The SOMOs of the first three ELMs are indicated by isosurfaces.

\section{B. Force field parameters}

The site energies $H_{k k}$ and corresponding forces $\nabla_{I} H_{k k}$ are calculated with a classical force field as described in Section III A. For the neutral ELM, we use the standard parameters taken from the Generalized Amber Force Field (GAFF) ${ }^{87}$ For the positively charged ELM, the excess charge of +1 is equally distributed over the two $\mathrm{C}$ atoms, and the van der Waals parameters are the ones of the neutral ELM. Intramolecular reorganization upon change of charge is modeled by a change in equilibrium distance between the two carbon bonds, all other bonding parameters remain unchanged. For simulation of the ELM dimer, the equilibrium bond length for the charged ELM was chosen so that the reorganization energy for hole transfer between the two ELMs at infinite separation,

$$
\lambda=\left[E_{\mathrm{C}}\left(\mathbf{R}_{\mathrm{N}}\right)+E_{\mathrm{N}}\left(\mathbf{R}_{\mathrm{C}}\right)\right]-\left[E_{\mathrm{C}}\left(\mathbf{R}_{\mathrm{C}}\right)+E_{\mathrm{N}}\left(\mathbf{R}_{\mathrm{N}}\right)\right],
$$

is $\lambda=0.3 \mathrm{eV}$. In Eq. (45), $E_{\mathrm{C}}\left(\mathbf{R}_{\mathrm{N}}\right)$ is the total force field energy of the charged ELM (subscript $\mathrm{C}$ ) at the minimum energy configuration of the neutral ELM, $\mathbf{R}_{\mathrm{N}}, E_{\mathrm{N}}\left(\mathbf{R}_{\mathrm{C}}\right)$ is the total force field energy of the neutral ELM (subscript N) at the minimum energy configuration of the charged ELM, $\mathbf{R}_{C}$, and $E_{\mathrm{C}}\left(\mathbf{R}_{\mathrm{C}}\right), E_{\mathrm{N}}\left(\mathbf{R}_{\mathrm{N}}\right)$ are the energies at the minimum energy configurations of the respective states. This gave a $\mathrm{C}=\mathrm{C}$ equilibrium bond length $r_{0}=1.401 \AA$ for the charged ELM using $r_{0}=1.324 \AA$ for the neutral ELM and a harmonic bond force constant of $589.7 \mathrm{kcal} \mathrm{mol}^{-1} \AA^{-2}$ from the GAFF force field. For the 10 ELM system, simulations demonstrating energy conservation were carried out for $\lambda=0.3 \mathrm{eV}$ and simulations of charge mobility for $\lambda=0.2 \mathrm{eV}$. For the latter, $r_{0}$ of the charged ELM was adjusted to $1.387 \AA$ while leaving all other parameters unchanged.

The off-diagonal elements $H_{k l}, k \neq l$, are calculated using the AOM method, Eq. (38), as described in Section III B. We treated the constant of proportion $C$ as a free parameter to investigate different coupling regimes. Simulations of the ELM dimer were carried out for $C=88.7 \mathrm{meV}$ and $443.5 \mathrm{meV}$ giving average coupling values $\left\langle\left|H_{12}\right|^{2}\right\rangle^{1 / 2}=8 \mathrm{meV}$ and $41 \mathrm{meV}$, respectively. Simulations of the 10 ELM system were carried out for $C=17.74,177.4$, and $1419 \mathrm{meV}$ giving average coupling values $\left\langle\left|H_{12}\right|^{2}\right\rangle^{1 / 2}=2.1 \mathrm{meV}, 21 \mathrm{meV}$, and $140 \mathrm{meV}$, respectively, which we refer to as small, medium, and high electronic coupling regimes. The calculation of the overlap integrals $S_{k l}$ was carried out as described in Ref. 65 using a minimum Slater basis of $p$ orbitals with Slater decay coefficients $\bar{\mu}_{2 p}=1.0000$ a.u. ${ }^{-1}$. The NACVs, and by virtue of Eqs. (41) and (42) $\nabla_{I} H_{k l}$ and $\nabla_{I} S_{k l}$, respectively, are obtained by numeric differentiation according to Eq. (44) using an increment $\Delta s=10^{-4} \AA$. For calculation of the hopping probability, the NACEs are calculated by numeric differentiation according to Eq. (40).

\section{FOB-SH simulations}

For the dimer system, a classical MD trajectory was generated, where the first ELM was modeled in the positively charged state and the other ELM in the neutral state. A weak harmonic position restraint with force constant of $1.0 \mathrm{kcal} \mathrm{mol}{ }^{-1} \AA^{-2}$ was applied to maintain a stable intermolecular separation of about $4 \AA$ between the ELMs. Configurations from this run were selected for FOB-SH simulations, with the initial electronic state chosen to be $\varphi_{1}$, i.e., the hole is fully localized on the first molecule. An electronic time step $\delta t=0.1 \mathrm{fs}$ was used, which was found to be the maximum possible time step without introducing errors into the charge carrier propagation for the two systems investigated. The nuclear time step was $\Delta t=0.5 \mathrm{fs}$. FOB-SH simulations were carried out in the NVT ensemble at $300 \mathrm{~K}$ using a Langevin thermostat with a friction constant $\gamma=10 \mathrm{ps}^{-1}$. A decoherence correction was applied as follows. In the model with 2 ELMs, with only two adiabatic surfaces, when the system was in the adiabatic ground state, the electronic wavefunction was collapsed onto the ground state 
when the system passed through one of the ground state minima in the direction away from the crossing point. In the model with 10 ELMs (see below), where the adiabatic potential energy surfaces are not analytically known in terms of the localized (site) basis states, the wavefunction was collapsed instead onto the current adiabatic state if the NACEs between the current state and all other states in the system fell below a threshold of $5 \times 10^{-6}$ a.u. $^{-1}$

For the calculation of charge mobility along the 1D chain of $M=10$ ELMs, a classical MD trajectory at temperature $T$ was generated for $1 \mathrm{~ns}$ without surface hops, where the first ELM is modeled in the positively charged state and the remaining 9 ELMs in the neutral state. A restraint potential is applied as described above. Configurations from that MD trajectory were taken as initial configurations and velocities for FOB-SH runs. Simulation parameters are the same as described above except where mentioned otherwise. For each FOB-SH trajectory, the mean-squared displacement (MSD) of the centre of charge of the carrier was obtained from the time evolution of the expansion coefficients in the site basis, Eq. (9), and averaged over all $N$ FOB-SH trajectories,

$$
\begin{aligned}
R^{2}(t) & =\frac{1}{N} \sum_{n=1}^{N}\left\langle\Psi_{n}(t)|x| \Psi_{n}(t)\right\rangle^{2} \\
& =\frac{1}{N} \sum_{n=1}^{N}\left(\sum_{l=1}^{M}\left|u_{l, n}(t)\right|^{2} x_{l, n}(t)\right)^{2},
\end{aligned}
$$

where $x_{l, n}$ is the position of the centre of mass of ELM $l$ in trajectory $n$, and $\sum_{l=1}^{M}\left|u_{l, n}(t)\right|^{2}=1$. The SH trajectories were initiated with the charge carrier localized on the first site, hence $R^{2}(0)=0$. When the MSD is linear, which was the case for the system investigated here, the Einstein diffusion constant $D$ can be obtained from the MSD,

$$
D=\frac{1}{2} \frac{\mathrm{d} R^{2}(t)}{\mathrm{d} t} .
$$

Eq. (48) is obtained by solving the 1D diffusion equation for the initial condition $\rho(x, t=0)=\delta(x)$ and zero flux boundary condition $\partial \rho /\left.\partial x\right|_{x=0, t}=0$, where $\rho$ is the probability density. Therefore Eq. (48) describes diffusion along the chain until the charge, initially at $x=0$, begins to populate the terminal site, i.e., in the short time limit. The mobility $\mu$ is then given by

$$
\mu=\frac{e D}{k_{B} T},
$$

where $e$ is the elementary charge and $k_{B}$ the Boltzmann constant. MSDs were calculated for small, medium, and high electronic coupling regimes (see Section IV B for ET parameters $\lambda$ and $C$ ) and for a wide range of temperatures, $T=50,75,100,150,200,250,300,500,800$, and $1000 \mathrm{~K}$. The MSD was averaged over 500 FOB-SH trajectories to obtain $R^{2}(t)$. In each case, there was a clear linear regime in the timeevolution of $R^{2}(t)$, occurring before charge carrier amplitude was building up at the final site of the system (which would introduce artificial reflection effects). $D$ was obtained from a linear fit to this region in each case. The statistical uncertainty of the mobility, obtained by block averaging of $R^{2}(t)$, was typically $10 \%$ of the mean value.

\section{Polaron hopping/kinetic Monte Carlo simulation}

In addition to FOB-SH simulations, the charge mobility of the 10 ELM system was also calculated assuming charge carrier hopping. The hopping rate from site $l$ to $k, l, k=1, \ldots, 10$, was obtained from the semi-classical expression $^{88-91}$

$$
k_{k l}=\kappa_{k l} v_{\mathrm{n}} \exp \left(-\frac{\max \left[\Delta A_{k l}^{\ddagger}, 0\right]}{k_{\mathrm{B}} T}\right),
$$

where $\kappa_{k l}=\kappa_{k l}\left(H_{k l}, \lambda, v_{n}\right)$ is the electronic transmission coefficient, $v_{n}$ is the effective nuclear frequency along the reaction coordinate, and $\Delta A_{k l}^{\ddagger}=(\lambda / 4)-\Delta_{k l}$ is the ET activation free energy with $\Delta_{k l} \sim H_{k l}$. For an explicit expression of $\kappa_{k l}$ and $\Delta_{k l}$, see, e.g., Ref. 92. We have chosen $v_{n}=1700 \mathrm{~cm}^{-1}$, representative of the $\mathrm{C}=\mathrm{C}$ stretch frequency, and $\lambda=0.2 \mathrm{eV}$ as in the FOB-SH simulations. According to Eq. (50), for configurations where $\Delta A_{k l}^{\ddagger}$ was negative as a consequence of large electronic coupling, the exponent was set to zero, corresponding to activation-less ET. Charge mobilities were calculated using an in-house written kinetic Monte Carlo (KMC) code. KMC trajectories were initiated from the same configurations from which the FOB-SH runs were initiated, and they were run for the same values of electronic coupling strength (i.e., values of $C$ ) and temperatures $T$ as the FOB$\mathrm{SH}$ simulations. For each initial configuration and values of $C$ and $T$, a number of KMC trajectories were generated, typically $1 \times 10^{5}-5 \times 10^{5}$ depending on the coupling strength. The mobility was calculated as $\mu=(\partial v / \partial E)_{E=0}$, where $v$ is the drift velocity of the charge carrier and $E$ is the electric field along the chain. The derivative was approximated by a 3-point finite difference calculation using an increment of $\delta E=10^{5} \mathrm{~V} \mathrm{~cm}^{-1}$. The drift velocity was obtained by averaging over all KMC trajectories and over all initial configurations with an applied bias of $\pm \delta E$ along the $x$ direction, aligned with the direction of the chain. The statistical uncertainty of the mobility obtained by block averaging of the drift velocity is typically $22 \%$ of the mean value.

\section{RESULTS AND DISCUSSION}

\section{A. Validation of implementation}

With the algorithm from Section II implemented as described in Section III, a validation of the numerical implementation is necessary. While the AOM method for calculation of the electronic Hamiltonian matrix elements $H_{k l}$ has been assessed before against DFT and wavefunction theory calculations $^{65}$ (summarized in Section III B), the calculation of the NACVs and the nuclear forces on the adiabatic potential energy surfaces are new features that need to be tested. Ultimately, the charge carrier dynamics predicted by FOB$\mathrm{SH}$ should be tested on small systems that are amenable to SH simulation with electronic structure calculations at the $a b$ initio level of theory ${ }^{35}$ or with TDDFT in combinational 
with a validated exchange correlation functional. Such investigations are planned for the future.

\section{Non-adiabatic coupling vectors: AOM vs FODFT}

Similar to the validation of $H_{k l}$ in Ref. 65, we assess the NACVs obtained from AOM against reference values at the FODFT level. In both cases the NACVs are calculated according to the finite difference approximation, Eq. (44). Test calculations were carried out on stacked ethylene dimers with various stacking distances of $3.5 \AA$, $4.5 \AA$, and $5.0 \AA$ and displacements of $\mathrm{C}$ atoms in the $x, y$, and $z$-directions, see Figure 2(a). In the FODFT calculations, the KohnSham orbitals of the molecules are fully re-optimized after each nuclear displacement $\delta s$ and the overlap between the DFT SOMOs calculated using grid integration. In the AOM calculations, where the DFT SOMOs are projected on a minimum-Slater type basis according to Eq. (37), we assume that the projection coefficients $c_{p \pi, i}$ are the same in the displaced and undisplaced structure and they were obtained for the minimum energy structures of the molecules in vacuum. Hence, comparison to FODFT reference values informs us about errors due to the projection of the DFT SOMO on the minimum Slater-type basis and the freezing of the projection coefficients.

Table I summarizes the results. The NACV component along the stacking $(x-)$ direction is very well reproduced with AOM. The absolute maximum error is $0.03 \AA^{-1}$, occurring at van der Waals contact at $3.5 \AA$. The agreement is excellent for the larger distances, $4.5 \AA$ and $5.0 \AA$. The NACV component along the $y$-direction is zero for both methods due to symmetry. The component parallel to the $\mathrm{C}=\mathrm{C}$ bond ( $z$-direction) showed a larger deviation with respect to the FODFT results. To see whether this is due to the freezing of the projection coefficients, we have carried out AOM calculations where projection coefficients are calculated for both displaced and undisplaced structures and subsequently

TABLE I. Non-adiabatic coupling vectors (NACVs) $\mathbf{d}_{I, k l}^{\prime}$, Eq. (44), between the charge localized states (SOMOs) of a stacked ethylene dimer from fragment-orbital DFT (FODFT) calculations, analytic overlap method without (AOM) and with update of expansion coefficients (AOM (updated)). ${ }^{\mathrm{a}}$

\begin{tabular}{llcc}
\hline \hline Separation & \multicolumn{1}{c}{ Method } & {$\left[\mathbf{d}_{\mathrm{CA} 1,21}^{\prime}\right]_{x}\left(\AA^{-1}\right)$} & {$\left[\mathbf{d}_{\mathrm{CA} 1,21}^{\prime}\right]_{z}\left(\AA^{-1}\right)$} \\
\hline $3.5 \AA$ & FODFT & $6.0 \times 10^{-2}$ & $4.0 \times 10^{-2}$ \\
& AOM & $3.4 \times 10^{-2}$ & $3.1 \times 10^{-3}$ \\
& AOM (updated) & $4.1 \times 10^{-2}$ & $5.8 \times 10^{-3}$ \\
\hline $4.5 \AA$ & FODFT & $2.2 \times 10^{-2}$ & $2.0 \times 10^{-2}$ \\
& AOM & $2.1 \times 10^{-2}$ & $1.2 \times 10^{-3}$ \\
& AOM (updated) & $1.7 \times 10^{-2}$ & $3.8 \times 10^{-3}$ \\
\hline $5.0 \AA$ & FODFT & $1.3 \times 10^{-2}$ & $1.4 \times 10^{-2}$ \\
& AOM & $1.5 \times 10^{-2}$ & $1.4 \times 10^{-3}$ \\
& AOM (updated) & $1.0 \times 10^{-2}$ & $2.7 \times 10^{-3}$ \\
\hline \hline
\end{tabular}

${ }^{\mathrm{a}}$ For each method, calculations were carried out at three different intermolecular separations as indicated. Due to symmetry, $\left[\mathbf{d}_{\mathrm{CB} 1,21}^{\prime}\right]_{x}=\left[\mathbf{d}_{\mathrm{CA} 1,21}^{\prime}\right]_{x},\left[\mathbf{d}_{\mathrm{CB} 1,21}^{\prime}\right]_{z}$ $=-\left[\mathbf{d}_{\mathrm{CA} 1,21}^{\prime}\right]_{z} \cdot\left[\mathbf{d}_{\mathrm{CA} 1,12}^{\prime}\right]_{x}=-\left[\mathbf{d}_{\mathrm{CA} 2,21}^{\prime}\right]_{x},\left[\mathbf{d}_{\mathrm{CA} 1,12}^{\prime}\right]_{z}=\left[\mathbf{d}_{\mathrm{CA} 2,21}^{\prime}\right]_{z}$ and similar for CB1, CB2. $\left[\mathbf{d}_{I, 21}^{\prime}\right]_{y}=\left[\mathbf{d}_{I, 12}^{\prime}\right]_{y}=0$ for all carbon atoms. See Figure 2 for definition of atom labels. used for NACV calculation. The results of these calculations are also shown in Table I (denoted AOM (updated)). The updating of the projection coefficients gives slightly improved results for the $z$-direction, but a significant discrepancy still remains. The reason for this is still unclear and is subject of ongoing investigations.

The NACVs are used for the calculation of the offdiagonal forces according to Eqs. (41) and (42). Here we briefly analyze the importance of these forces. The largest NACV values are found for the stacked ethylene dimer at van der Waals contact, $6.0 \times 10^{-2} \AA^{-1}$ and $4.0 \times 10^{-2} \AA^{-1}$ in the $x$ - and $z$-directions, translating to an off-diagonal force of $2 \times 10^{-3}$ and $1 \times 10^{-3}$ Hartree/bohr, respectively (using $C=1819 \mathrm{meV}$ from Ref. 65). On both $\mathrm{C}$ atoms, the component in the stacking $(x-)$ direction points in the direction of the other monomer pushing them together or apart. This force can become comparable to the weak van der Waals forces between the molecules and should be included. On the other hand, the component in the $z$-direction along the $\mathrm{C}=\mathrm{C}$ bond is typically two orders of magnitude smaller than those arising from the bond force constant in the force field. In view of this analysis, the error in the force along the $z$-direction due to the error in the NACV can be neglected.

\section{Nuclear forces and energy conservation}

As demonstrated in Section II, for a dimer system, the nuclear forces on the active potential surface can be calculated using either Eq. (36) or the general $N$-site expression Eq. (21) for $N=2$. While both expressions are of course equivalent for a dimer, the implementation of the general $N$-site expression is more involved than the one for Eq. (36). A comparison between the numerical results obtained from the two expressions is therefore a good test for the validity of our implementation of Eq. (21). To achieve this, we carried out four FOB-SH simulations of 100 ps in length each, though with surface hops always being rejected. Hence, each simulation was carried out on a single adiabatic potential energy surface (ground or excited) and the scaling constant in Eq. (38) was chosen to produce two different values of the average electronic coupling, $\left\langle\left|H_{12}\right|^{2}\right\rangle^{1 / 2}=8 \mathrm{meV}$ and $41 \mathrm{meV}$, respectively. Thus, for two different values of the coupling, we produced both a ground-state and an excited-state trajectory. For each trajectory, at 100 regularly spaced configurations 1 ps apart, we calculated the forces on each atom according to both Eqs. (36) and (21), as well as a version of Eq. (21) that sets the off-diagonal forces $\nabla_{I} H_{k l}$ to 0 . This last expression was chosen to demonstrate how the importance of these off-diagonal forces changes depending on the strength of the coupling.

The results are shown in Figure 4, which plots the forces from the full expression Eq. (21) (symbols in red) and from Eq. (21) with $\nabla_{I} H_{k l}=0$ (symbols in green) against the ones obtained from the full expression Eq. (36). We observe exact agreement between Eqs. (21) and (36) for all four cases, validating the force calculation. In contrast, an error is made when neglecting the off-diagonal forces in Eq. (21), which becomes pronounced in the larger coupling case (panels A and C) and also is greatest on the excited state trajectory, where 


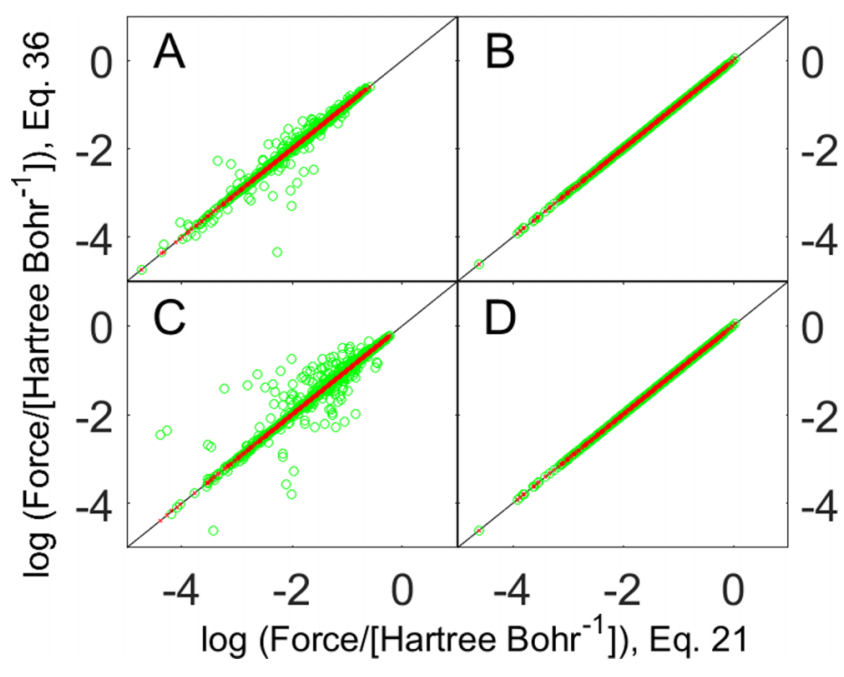

FIG. 4. Nuclear forces obtained from the general $M$-state expression, Eq. (21), and from the 2-state expression, Eq. (36). The $x, y$, and $z$ components of the nuclear forces in the ELM donor-acceptor complex are plotted for configurations obtained from MD simulation (a) on the adiabatic ground state $E_{0},\left\langle\left|H_{12}\right|^{2}\right\rangle^{1 / 2}=41 \mathrm{meV}$, (b) on the adiabatic ground state $E_{0}$, $\left\langle\left|H_{12}\right|^{2}\right\rangle^{1 / 2}=8 \mathrm{meV}$, (c) on the excited state $E_{1},\left\langle\left|H_{12}\right|^{2}\right\rangle^{1 / 2}=41 \mathrm{meV}$, and (d) on the excited state, $\left\langle\left|H_{12}\right|^{2}\right\rangle^{1 / 2}=8 \mathrm{meV} . \lambda=0.3 \mathrm{eV}$ in each case. Red crosses refer to the full force calculation in Eq. (21) while green circles refer to the diagonal force approximation $\left(\nabla_{I} H_{k l}=0\right)$, as described in Table II.

the relative effect of the off-diagonal force terms is maximum (panel C, up to 0.1 Hartree/bohr).

Having validated our force calculations and begun to understand the importance of the off-diagonal forces, we wanted to further investigate if our SH simulations would conserve total energy well, and to understand if the omission of off-diagonal gradients would cause a significant computational speedup. To see this, we performed individual FOB-SH simulations for the higher coupling strength model of between 0.1 and $1 \mathrm{~ns}$ in length for six different simulation setups in the NVE ensemble. Three of these were on model ELM dimers, respectively, calculating the nuclear forces on the active potential energy surface using Eq. (36), Eq. (21), and the version of Eq. (21) that sets the off-diagonal forces to 0 . The latter two force expressions were also applied to the 1D chain of 10 ELMs. For reference, we have also carried out a simulation on the electronic ground state with surface hops being suppressed. Table II summarizes the

TABLE II. Total energy conservation of FOB-SH simulation for the ELM dimer $\left(\lambda=0.3 \mathrm{eV},\left\langle\left|H_{\mathrm{ab}}\right|^{2}\right\rangle^{1 / 2}=41 \mathrm{meV}\right)$ and for a chain of 10 ELMs in the NVE ensemble $\left(\lambda=0.3 \mathrm{eV},\left\langle\left|H_{\mathrm{ab}}\right|^{2}\right\rangle^{1 / 2}=41 \mathrm{meV}\right)$, and the corresponding timing on a single compute core.

\begin{tabular}{lccc}
\hline \hline Force expression & Number of sites & $\begin{array}{r}\text { Total energy drift } \\
(\text { Hartree/ps/atom) }\end{array}$ & $\begin{array}{r}\text { CPU time } \\
(\mathrm{s} / \mathrm{ps})\end{array}$ \\
\hline Eq. (36), no hops & 2 & $1.78 \times 10^{-9}$ & 0.6 \\
Eq. (36) & 2 & $1.18 \times 10^{-6}$ & 31 \\
Eq. (21) & 2 & $8.75 \times 10^{-7}$ & 45 \\
Eq. (21), $\nabla_{I} H_{k l}=0$ & 2 & $7.40 \times 10^{-6}$ & 33 \\
Eq. (21) & 10 & $5.45 \times 10^{-6}$ & 1028 \\
Eq. (21), $\nabla_{I} H_{k l}=0$ & 10 & $1.04 \times 10^{-5}$ & 675 \\
\hline \hline
\end{tabular}

energy conservation and computational cost of each of these simulations.

We find that the total energy drift of our FOB-SH implementation is reasonably small, $10^{-6}$ Hartree/ps/atom for the 2 and 10 ELM systems. Yet, this is about 3 orders of magnitude higher than for the reference MD simulations without hops, $10^{-9}$ Hartree/ps/atom. The higher energy drift in the FOB-SH runs is related to the discontinuity in the forces that comes from undergoing surface hops. Tully's original publication $^{25}$ discussed this to some extent, choosing to keep the sudden switches for the sake of simplicity while noting suggestions for fictitious forces that would carry the molecules smoothly from one trajectory to another. We have also chosen to allow the system to hop suddenly between surfaces, both for reason of computational simplicity and also to avoid any possible outcome of the molecules being stuck on some interpolation between actual adiabatic surfaces while the system undergoes several surface hops.

The energy drift obtained for Eqs. (36) and (21) is very similar, which is expected as these two expressions are equivalent for $N=2$. When neglecting the off-diagonal gradients, the energy drift increases by about an order of magnitude, from $10^{-6}$ to $10^{-5}$ Hartree/ps/atom. This is consistent with the increasing error in the forces as shown in Figures 4(a) and 4(c). An energy drift of about $10^{-5}$ Hartree/ps/atom is typical for density functional-based molecular dynamics simulation, where much of the drift comes from the iteration of the Kohn-Sham equations in each time step. Hence, it appears that omission of the off-diagonal gradients still gives reasonable results for the coupling regime investigated here (a few $10 \mathrm{meV}$, typical for OS materials). However, the energy drift could quickly deteriorate further, in particular when surface hops occur more frequently. Therefore, we recommend that off-diagonal gradients should be included in FOB-SH simulation for OS materials. Finally, considering the timing in Table II, we highlight the computational efficiency of our implementation, allowing us to sample several picoseconds of $\mathrm{SH}$ dynamics per hour and CPU core for the 10 ELM model.

\section{B. FOB-SH for ELM dimer}

In the following, we consider the ELM dimer and analyze the mechanism of thermally activated hole transfer as obtained from FOB-SH. Before we do so, we need to define a few quantities. First, we define an initial charge-localized (diabatic) CT state A, where ELM 1 and ELM 2 are described with the force field topology of the positively charged and neutral states, respectively, with total potential energy (or site energy or diabatic energy) $E_{\mathrm{A}}(\mathbf{R}) \equiv H_{11}(\mathbf{R})$, where $\mathbf{R}$ is the nuclear configuration of the ELM dimer. Similarly, a final charge-localized state B is defined, where ELM 2 now carries the positive charge and ELM 1 is neutral, with site energy $E_{\mathrm{B}}(\mathbf{R}) \equiv H_{22}(\mathbf{R})$. Analogously with ET theory, we choose the vertical energy gap for transfer of the total charge from ELM 1 to ELM 2 at fixed nuclear configuration as the reaction coordinate,

$$
\Delta E(\mathbf{R})=E_{\mathrm{B}}(\mathbf{R})-E_{\mathrm{A}}(\mathbf{R}) .
$$


The reorganization energy was chosen to be $\lambda=0.3 \mathrm{eV}$ and the coupling strength was chosen to be $C=88.7 \mathrm{meV}$ as before, giving $\left\langle\left|H_{12}\right|^{2}\right\rangle^{1 / 2}=8 \mathrm{meV}$. In this regime, the adiabatic ground state potential energy exhibits a double well feature (see Figure 1) with an activation barrier that is sufficiently low so that thermal CT is expected to occur on the $100 \mathrm{fs}$ to ps time scale $\left(\Delta E^{\ddagger}=67 \mathrm{meV}\right)$. In the region around the left minimum, the adiabatic ground state surface $E_{0}(\mathbf{R})$ is almost identical with $E_{\mathrm{A}}(\mathbf{R})$ and in the right minimum with $E_{\mathrm{B}}(\mathbf{R})$.

The FOB-SH runs are initiated on the adiabatic ground state surface $E_{0}(\mathbf{R})$ using initial configurations that were sampled in $\mathrm{MD}$ simulations on the potential energy surface $E_{\mathrm{A}}(\mathbf{R})$ (hole localized on ELM 1). The initial hole wavefunction has an amplitude only on ELM 1, $u_{1}(0)=1$, $u_{2}(0)=0$. Since $E_{0}(\mathbf{R}) \approx E_{\mathrm{A}}(\mathbf{R})$ in the minimum region of A, the energy gap $\Delta E(\mathbf{R})$ shown in Figure 5(a) initially oscillates around the minimum of state $A$, that is, around $\Delta E=\lambda=0.3 \mathrm{eV}$. Thermal fluctuations in the site energy difference $\Delta E$ continually bring the system close to the crossing point ("resonance region") $\Delta E=0$, until a surface hop occurs at about 300 fs and another two follow at about $400 \mathrm{fs}$. During the lifetime of these excitations (a few $10 \mathrm{fs}$ or $\mathrm{C}=\mathrm{C}$ vibrations), the site energy difference is oscillating around $\Delta E=0$, which enables transfer of electronic hole population from ELM 1 to ELM 2 within about 300 fs. Eventually, the system settles down into the adiabatic ground state again, now in the minimum of the final diabatic state $\mathrm{B}$, and all of the hole amplitude is transferred to ELM 2, $u_{1}(t)=0, u_{2}(t)=1$ from $t=600$ fs.

The hole transfer taking place at around $\Delta E=0$ fits with what we expect from well known theory. If we consider a quantum system described by a time-independent Hamiltonian with two diabatic states $a$ and $b$, initialized with $P_{a}(0)=1, P_{b}(0)=0$. Then

$$
P_{b}(t)=\frac{4 H_{a b}^{2}}{\left(E_{b}-E_{a}\right)^{2}+4 H_{a b}^{2}} \sin ^{2}\left(\frac{1}{2} \Omega_{R} t\right),
$$

where $\Omega_{R}$ is the Rabi frequency, given by

$$
\Omega_{R}=\frac{1}{\hbar} \sqrt{\left(E_{b}-E_{a}\right)^{2}+4 H_{a b}^{2}} .
$$

Hence, significant CT can occur only when $|\Delta E|$ is on the order of $\left|H_{a b}\right|$ or less, with a maximum amplitude of

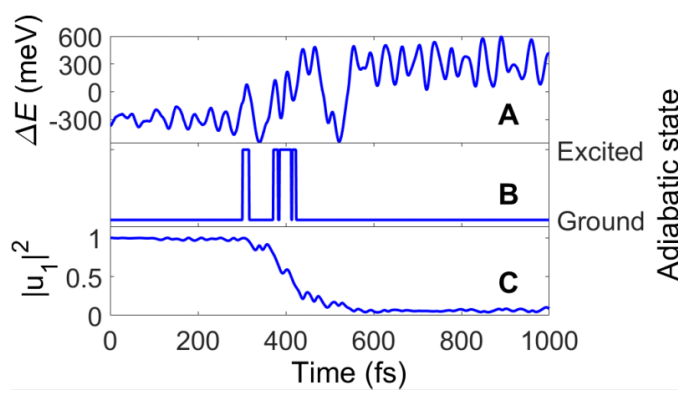

FIG. 5. Mechanism of hole transfer in the ELM donor-acceptor complex from FOB-SH simulation. Time series of (a) the diabatic energy gap $\Delta E$, Eq. (51), (b) the active adiabatic surface that determines the nuclear dynamics, either ground or excited, (c) the amplitude of the charge localized electronic hole state (SOMO) that is initially occupied. population transfer when $\Delta E=0$. The topology of the excited state $E_{1}(\mathbf{R})$ in the ELM dimer is such that it has a minimum at $\Delta E=0$. Hence, when the ELM dimer is on the excited state surface, the energy gap oscillates around $\Delta E=0$, which permits large transfer of hole amplitude between the ELMs.

We further analyzed the statistics of surface hops, by taking advantage of our efficient simulation approach to produce 500 trajectories for the ELM dimer with a total accumulated length of $1 \mathrm{~ns}$. In particular, we were interested to understand how the surface-hopping probability Eq. (4) correlates with the vertical energy gap $\Delta E$. One would usually expect that the probabilities are highest at the crossing region at $\Delta E=0$ and fall away to 0 rather quickly away from this point: this is due to the non-adiabatic coupling element between the surfaces being strongest at the crossing region and weakest away from it. Our results are shown in Figure 6: it is immediately clear that the behavior of the hopping probability is more complicated than originally envisaged. While we observe a clear peak of the distribution centred on $\Delta E=0$, we also observe configurations close to the crossing point with a vanishing hopping probability, e.g., when the NACVs are close to being orthogonal to the nuclear velocities. What is perhaps surprising is that a small but finite hopping probability survives into the initial and final state minima at $\pm 300 \mathrm{meV}$. Hence, in a small number of cases, we observe surface hops when the system is in one of the stable ground state potential energy minima.

\section{Charge mobility from FOB-SH and polaron hopping/KMC}

As a major motivation behind the development of this method was the ability to apply it to larger systems to study CT, we now turn to an application to the chain of 10 ELMs. We used this system to calculate the hole mobility along its length for varying values of temperature and electronic coupling strength. Our intention was to demonstrate that, similarly to a recent investigation by Wang and Beljonne, ${ }^{8}$ our FOB-SH method is capable of reproducing the thermally activated CT at low electronic coupling strength and its absence for higher coupling strength. We chose a range of temperature values

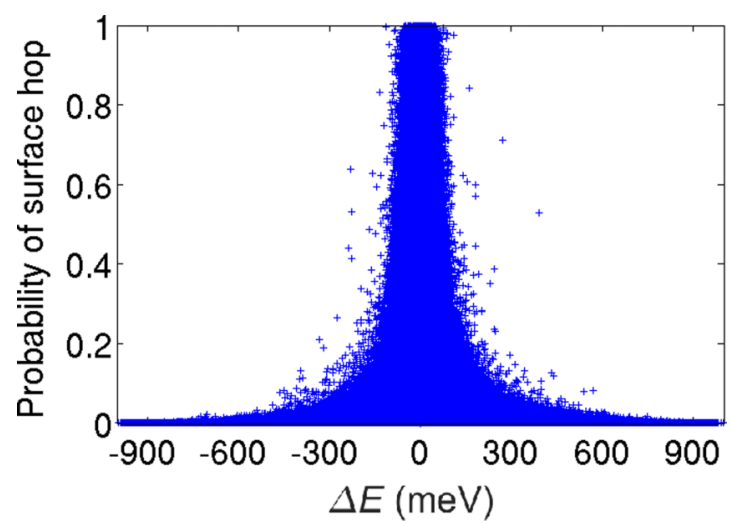

FIG. 6. Hopping probability Eq. (4) versus diabatic energy gap $\Delta E$, Eq. (51). Data are obtained from 500 FOB-SH runs for the ELM donor-acceptor complex with a total accumulated length of $1 \mathrm{~ns}$. 
for three different values of electronic coupling strength, see Section IV C for simulation details. The mobilities obtained from FOB-SH according to Eq. (49) are plotted against temperature in Figure 7.

As we anticipated, we can see a thermally activated charge transport for the smallest electronic coupling strength (blue symbols) at low temperatures. Around room temperature, we observe a crossover from activated to band-like transport with the mobility decreasing for increasing temperatures according to $\mu \propto T^{-3.5}$. With increasing electronic coupling strengths, thermal activation gradually disappears. For the highest coupling strength investigated (red symbols), the mobility decreases steadily with $T$ according to $\mu \propto T^{-1.2}$. A qualitatively very similar behavior, i.e., crossover from activated to band-like transport at low coupling and disappearance of activated regime at high coupling regime, has been obtained from $\mathrm{SH}$ simulations by Wang and Beljonne. ${ }^{8}$ Moreover, an inverse power law dependence of the mobility with temperature similar to the one observed here for the large coupling regime has been reported by Troisi and Orlandi6,54 and by Fratini et al. ${ }^{3}$

To explain the steady decrease in mobility with increasing temperature in the high coupling regime, we return to the concept of resonance for charge transfer. In Figure 8(a), we plot the probability distribution of the vertical energy gap between the first site where the charge is initially located and the second site, $\Delta E_{12}$, along an equilibrium MD simulation in that initial electronic state, for different temperatures. We also indicate an averaged resonance region arising from the mean electronic coupling along these trajectories, $\left\langle\left|H_{12}\right|\right\rangle=129.9 \mathrm{meV}$, which remains rather insensitive on temperature. For low temperature, we find that the peak of the thermal equilibrium distribution of $\Delta E_{12}$ is already well within the resonance region, which we define with the boundaries $\Delta E_{12}= \pm 2\left\langle\left|H_{12}\right|\right\rangle$, as at these boundaries the prefactor in Eq. (52) is exactly 0.5 and potentially allows half the charge to transfer to the second site. Therefore propagation of the charge carrier occurs instantly for the vast majority of initial configurations. A temperature increase will broaden the

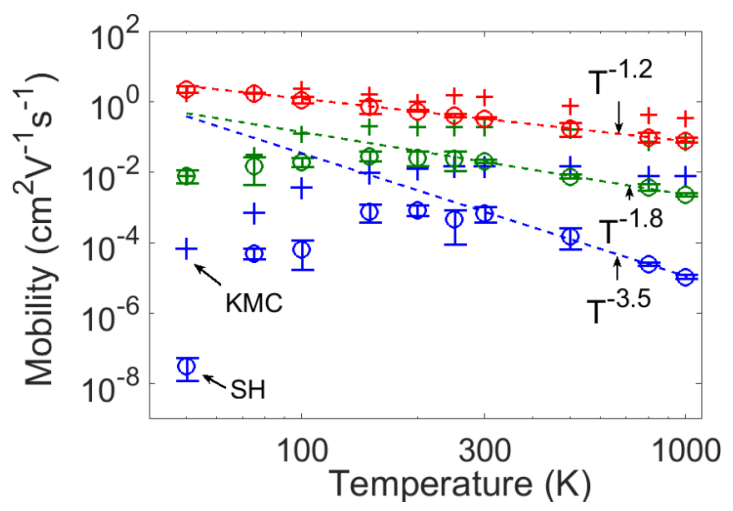

FIG. 7. Temperature dependence of hole mobility along a chain of 10 ELMs, for three different values of average electronic coupling: $\left\langle\left|H_{12}\right|^{2}\right\rangle^{1 / 2}$ $=2.1 \mathrm{meV}$ (blue), $\left\langle\left|H_{12}\right|^{2}\right\rangle^{1 / 2}=21 \mathrm{meV}$ (green), $\left\langle\left|H_{12}\right|^{2}\right\rangle^{1 / 2}=140 \mathrm{meV}$ (red), $\lambda=0.2 \mathrm{eV}$ in each case. Results from FOB-SH (SH, $\bigcirc$ symbols) and for small polaron hopping/kinetic Monte Carlo simulation (KMC, + symbols) are shown. Best fits of the FOB-SH data between 300 and $1000 \mathrm{~K}$ to an inverse power-law are indicated by dashed lines.
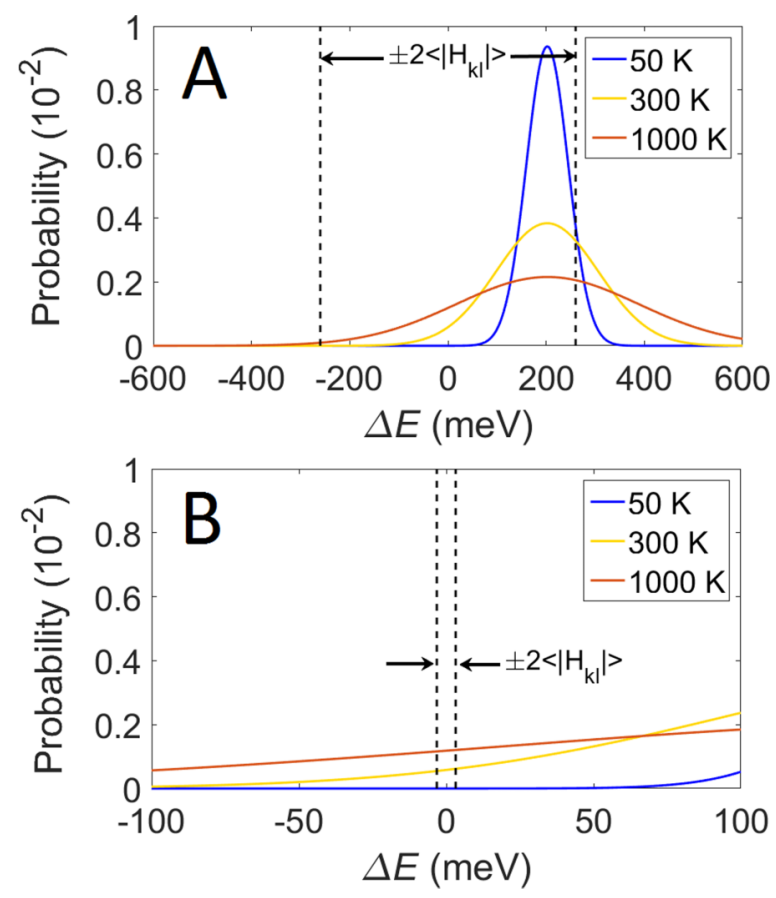

FIG. 8. Normalized probability distributions of the site energy difference $\Delta E_{12}$ between first and second ELM obtained from an equilibrium trajectory for the 10 ELM chain with the hole localized on the first ELM. Data are shown for three different temperatures within the range plotted in Figure 7. The resonance region for $\mathrm{CT}$ is indicated by arrows. As the temperature increases, thermal fluctuations in the site energies broaden these distributions. For sufficiently high values of $\left\langle\left|H_{12}\right|\right\rangle$, as shown in panel $\mathrm{A}$, this reduces the area under the curve that is within the resonance region and the mobility thus decreases accordingly with temperature. For smaller values of $\left\langle\left|H_{12}\right|\right\rangle$, as shown in panel $\mathrm{B}$, this broadening instead allows the area under the curve in the resonance region to increase from zero and thus increasing temperature instead increases mobility.

distribution for $\Delta E_{12}$ and thereby reduces the probability for the system to be within the resonance region. Consequently, the time it takes for the charge to move from site 1 to site 2 and further along the chain will increase and hence the mobility will decrease.

The situation is strikingly different for the temperatureactivated transport observed in the low coupling regime, Figure $8(b)$. Here the peak of the equilibrium distribution of $\Delta E_{12}$ is outside the very narrow resonance region $\left(\left\langle\left|H_{12}\right|\right\rangle=1.6 \mathrm{meV}\right)$. As the temperature increases, the energy gap distribution becomes wider and the probability for resonance increases. Therefore the charge mobility increases with increasing temperature at low temperature. The reason for the crossover at about room temperature is less clear, though we think that the effect of increasing probability for resonance saturates at a sufficiently high temperature so that the $T^{-1}$ term of Eq. (49) takes over. The observed $T^{-3.5}$ dependence points to an additional effect that leads to a decrease of the diffusion constant with temperature in this regime.

Our explanation of the $T$-dependence of mobility is further illustrated in Figure 9, where we plot the fraction of time (= probability) that the system has spent in the resonance region $\Delta E_{12}(t)= \pm 2\left|H_{12}(t)\right|$ during the same equilibrium MD runs described above (first site in charged state, all other sites in neutral state). We assert that this resonance probability be proportional to the diffusion constant and in the spirit of 


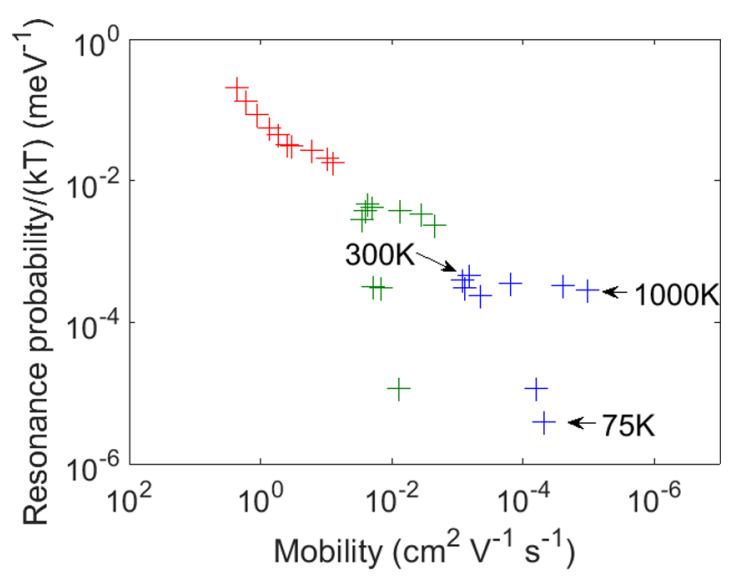

FIG. 9. Correlation between the resonance probability for the first two molecules in the ELM chain divided by $k_{\mathrm{B}} T$, and the corresponding hole mobility when the temperature is changed. Mobilities are shown for the three different coupling regimes defined in Figure 7 (same color code applies). The mobilities are taken from Figure 7.

Eq. (49) divide it by $k_{B} T$ to correlate it with the mobility from $\mathrm{SH}$. As one can see in Figure 9, the correlation is reasonably good for both band-like and thermally activated regimes.

For comparison, we have also plotted in Figure 7 mobilities calculated for the polaron hopping model using KMC simulation, as explained in Section IV D. For the lowest coupling regime, the KMC demonstrates a thermal activation at low $T$ due to the presence of small activation barrier, but a much weaker mobility decay than FOB-SH at high $T$. In the high coupling regime, the mobility decay with temperature is underestimated compared to FOB-SH. We note that Eq. (50), which the hopping model relies upon for rate calculations, is problematic for the high coupling regime due to the absence of an energy barrier for charge transfer. Interestingly, below room temperature, the mobility from hopping and FOB-SH are about the same. Therefore, care needs to be taken when comparing the two approaches-any agreement between them may be fortuitous and should be scrutinized further, e.g., by determining the $T$-dependence of the mobility at the temperature of interest.

\section{CONCLUSIONS}

We have successfully implemented a very efficient surface hopping algorithm, denoted fragment orbital-based surface hopping (FOB-SH), that is designed for the simulation of charge carrier transport in large pi-conjugated systems such as OS materials and biological molecules. A key feature is the construction of the electronic Hamiltonian in a basis of fragment orbitals (SOMOs) of the molecules or molecular fragments. We have derived a general expression for the forces on the adiabatic potential energy surfaces in terms of nuclear gradients of the charge localized states, and we have validated our force expression and have demonstrated its use, with applications on a simple donor-acceptor system as well as a larger one-dimensional chain. With both of these systems we have demonstrated our method's ability to investigate relevant CT properties.
For the chain of 10 ELMs, we observed a crossover from activated to band-like charge transport for small electronic couplings. The activated regime gradually disappears with increasing electronic coupling resulting in an inverse powerlaw dependence of mobility with temperature. This is in qualitative agreement with previous SH simulations by Wang and Beljonne for $\mathrm{CT}$ along a chain of harmonic oscillators. ${ }^{8}$ A power-law decay was also observed by $\mathrm{Troisi}^{55}$ for single crystalline rubrene, which falls in the large coupling regime investigated here. It is important to note that our results are obtained without assuming a specific charge transport model: the CT mechanism is a result of the FOB-SH simulation using only the CT mediating SOMOs as an input. The calculation of charge mobility on a many-molecule one-dimensional system was a good proof of concept and demonstrates how the method shows promise for tackling future applications for two and three-dimensional systems.

Similarly to the method of Kubař and Elstner, ${ }^{44,45}$ our FOB-SH methodology bridges the gap between the semiempirical SH implementations ${ }^{5,46,47,49,51}$ and the harmonic oscillator/model Hamiltonian approaches developed by Troisi and Orlandi ${ }^{6,54}$ and by Wang and Beljonne. ${ }^{8}$ Like semiempirical models, it is a fully atomistic MD method but, at variance with them, only the $\mathrm{CT}$ mediating redox active orbital(s) of each molecule or molecular fragment are treated by a quantum mechanical parametrization, whereas the rest of the molecule is treated with a classical force field. This makes the FOB-SH method highly efficient. It also offers several advantages with respect to harmonic oscillator/model Hamiltonian approaches: (i) "sites" are molecules in full atomistic representation in FOB-MD rather than one-dimensional harmonic oscillators; (ii) parameters related to local and non-local electron-phonon coupling in harmonic oscillator models are generated on-the-fly during FOB-SH and are time-dependent rather than constants; (iii) the quantum back reaction from the electronic to the nuclear degrees of freedom is included allowing, e.g., the description of spontaneous polaron formation/charge localization; and (iv) the effect of the environment on site energy and electronic coupling fluctuations can be included at the force field level. Therefore, the FOB-SH method is expected to give a realistic picture of $\mathrm{CT}$ in organic materials at a computational cost similar to those of classical MD simulations.

The method presented here is in its early stages, and we foresee many avenues of improvement still to come. Although we believe that our electronic Hamiltonian is a good model, there remains the possibility to increase the accuracy of the calculation of both diagonal and off-diagonal elements of it. At present the diagonal elements of the Hamiltonian are obtained with a fixed point charge model. We are presently working on an expansion of our current implementation which will enable the calculation of the site energies with polarizable force fields, improving the method's ability to model the polarizable environment. We may also be able to increase the accuracy of the off-diagonal Hamiltonian matrix elements and NACVs further. Interpolation schemes or machine learning techniques could be used to better reconstruct the SOMO along the FOB-SH trajectory and a larger basis set could be used to improve their representation. 
There are also ways in which the surface hopping algorithm can be improved. We have discussed how a correction to the electronic over-coherence problem is necessary to match the system's long-term behavior to physical intuition, but our current decoherence correction is relatively crude, with a simple collapse of the electronic wavefunction whenever the system meets a certain criterion. The effect of the decoherence correction and discussions of different approaches are common in the literature, as we have referenced in Section III G: it has been shown that it is necessary to treat the effects of electronic over-coherence not just to avoid nonsensical system behavior at long time scales but also, e.g., to obtain a correct statistical balance of adiabatic state populations. Obvious expansions to our method would therefore include a more thorough treatment of this decoherence problem, and additionally an algorithm capable of detecting and handling so-called trivial, or non-avoided, crossings. ${ }^{8,93}$ This latter problem needs to be accounted for in larger systems, where adiabatic electronic states may be spatially distant (non-interacting) but very close in energy. Thus this issue will also need to be addressed if the method is to be applied, as we intend, to realistic model systems for OS materials.

Moving to larger systems also motivates us to search for even greater computational efficiency. Although the method as it stands is extremely fast compared to standard electronic structure methods, each simulation is run on a single computational core and a significant speedup could in theory be obtained by parallelizing the method. We anticipate that the calculation of the Hamiltonian elements, in particular, the analytic overlap method (which currently accounts for the majority of the time for each simulation), could be parallelized to both reduce simulation time and improve the scaling with system size. Parallelizing the site energy calculations would also allow us to offset the loss of computational efficiency that would come with implementing a more advanced force field.

The method presented in this paper shows promise for applications to a variety of condensed phase systems, both as it currently exists and with the planned improvements we list above. In future work we intend to use it on CT problems in organic molecular crystals and disordered systems such as might be found in organic electronic devices, and on ultrafast electron transport problems in solvated biomolecules such as proteins and DNA. The computational efficiency of the method should make it possible to carry out these simulations with the required system size and time scales, providing significant insight into important current research questions of charge transport in aqueous, organic, and biological environments.

\section{ACKNOWLEDGMENTS}

We would like to thank Antoine Carof for making us aware of Eqs. (30)-(33) and for helpful comments on this manuscript. Laura Scalfi is acknowledged for her help with debugging the computer code. J.S. was supported by an IMPACT Ph.D. studentship co-sponsored by University College London and the Department of Physics and Astronomy. The authors acknowledge the use of the UCL High Performance Computing Facility "Legion" and associated support services.

${ }^{1}$ V. Coropceanu, J. Cornil, D. A. da Silva, Y. Olivier, R. Silbey, and J.-L. Bredas, Chem. Rev. 107, 926 (2007).

${ }^{2}$ J. Nelson, J. J. Kwiatkowski, J. Kirkpatrick, and J. M. Frost, Acc. Chem. Res. 42, 1768 (2009).

${ }^{3}$ S. Fratini and S. Ciuchi, Phys. Rev. Lett. 103, 266601 (2009).

${ }^{4}$ F. Ortmann, F. Bechstedt, and K. Hannewald, Phys. Rev. B 79, 235206 (2009).

${ }^{5}$ M. J. Bedard-Hearn, F. Sterpone, and P. J. Rossky, J. Phys. Chem. A 114, 7661 (2010)

${ }^{6}$ A. Troisi, Chem. Soc. Rev. 40, 2347 (2011).

${ }^{7}$ J. Ren, N. Vukmirovic, and L.-W. Wang, Phys. Rev. B 87, 205117 (2013).

${ }^{8}$ L. Wang and D. Beljonne, J. Phys. Chem. Lett. 4, 1888 (2013).

${ }^{9}$ A. V. Akimov and O. V. Prezhdo, J. Am. Chem. Soc. 136, 1599 (2014).

${ }^{10}$ A. Heck, J. J. Kranz, T. Kubar̆, and M. Elstner, J. Chem. Theory Comput. 11, 5068 (2015).

${ }^{11}$ D. L. Cheung and A. Troisi, J. Phys. Chem. C 114, 20479 (2010).

${ }^{12}$ H. Oberhofer and J. Blumberger, Phys. Chem. Chem. Phys. 14, 13846 (2012).

${ }^{13} \mathrm{~F}$. Gajdos, H. Oberhofer, M. Dupuis, and J. Blumberger, J. Phys. Chem. Lett. 4, 1012 (2013)

${ }^{14}$ M. E. Gershenson, V. Podzorov, and A. F. Morpurgo, Rev. Mod. Phys. 78, 973 (2006)

${ }^{15}$ F. C. Grozema and L. D. A. Siebbeles, Int. Rev. Phys. Chem. 27, 87 (2008).

${ }^{16}$ J. Ide, D. Fazzi, M. Casalegno, S. V. Meille, and G. Raos, J. Mater. Chem. C 2, 7313 (2014)

${ }^{17}$ G. A. Worth, H.-D. Meyer, H. Koppel, L. S. Cederbaum, and I. Burghardt, Int. Rev. Phys. Chem. 27, 569 (2008).

${ }^{18}$ H. Tamura and I. Burghardt, J. Am. Chem. Soc. 135, 16364 (2013).

${ }^{19}$ T. J. Martinez, M. Ben-Nun, and R. Levine, J. Chem. Phys. 100, 7884 (1996).

${ }^{20}$ T. J. Martinez, Acc. Chem. Res. 39, 119 (2006)

${ }^{21}$ A. Abedi, N. T. Maitra, and E. K. U. Gross, Phys. Rev. Lett. 105, 123002 (2010).

${ }^{22}$ A. Abedi, N. T. Maitra, and E. K. U. Gross, J. Chem. Phys. 137, 22A530 (2012).

${ }^{23}$ F. Agostini, A. Abedi, and E. K. U. Gross, J. Chem. Phys. 141, 214101 (2014).

${ }^{24}$ P. Ehrenfest, Z. Phys. 45, 455457 (1927).

${ }^{25}$ J. C. Tully, J. Chem. Phys. 93, 1061 (1990)

${ }^{26} \mathrm{~J}$. C. Tully, in Classical and Quantum Dynamics in Condensed Phase Simulations, edited by B. J. Berne, G. Ciccotti, and D. F. Coker (World Scientific, 1998), pp. 34-71.

${ }^{27}$ J. C. Tully, Mixed Quantum-Classical Dynamics, Modern Methods for Multidimensional Dynamics Computations in Chemistry (World Scientific, 1998).

${ }^{28}$ G. Granucci and M. Persico, J. Chem. Phys. 126, 134114 (2007).

${ }^{29}$ J. C. Tully, J. Chem. Phys. 137, 22A301 (2012).

${ }^{30}$ A. V. Akimov, A. J. Neukirch, and O. V. Prezhdo, Chem. Rev. 113, 4496 (2013).

${ }^{31}$ L. Wang, O. V. Prezhdo, and D. Beljonne, Phys. Chem. Chem. Phys. 17, 12395 (2015).

${ }^{32}$ F. Plasser and H. Lischka, J. Chem. Phys. 134, 034309 (2011).

${ }^{33}$ R. Mitrić, V. Bonačić-Koutecký, J. Pittner, and H. Lischka, J. Chem. Phys. 125, 024303 (2006).

${ }^{34}$ L. Blancafort, P. Hunt, and M. A. Robb, J. Am. Chem. Soc. 127, 3391 (2005).

${ }^{35}$ F. Plasser, G. Granucci, J. Pittner, M. Barbatti, M. Persico, and H. Lischka, J. Chem. Phys. 137, 22A514 (2012).

${ }^{36}$ C. F. Craig, W. R. Duncan, and O. V. Prezhdo, Phys. Rev. Lett. 95, 163001 (2005).

${ }^{37}$ E. Tapavicza, I. Tavernelli, and U. Röthlisberger, Phys. Rev. Lett. 98, 023001 (2007).

${ }^{38}$ B. F. Curchod, T. J. Penfold, U. Rothlisberger, and I. Tavernelli, Cent. Eur. J. Phys. 11, 1059 (2013).

${ }^{39}$ N. L. Doltsinis and D. Marx, Phys. Rev. Lett. 88, 166402 (2002).

${ }^{40}$ N. L. Doltsinis and D. Marx, J. Theor. Comput. Chem. 1, 319 (2002).

${ }^{41}$ M. Elstner, D. Porezag, G. Jungnickel, J. Elsner, M. Haugk, T. Frauenheim, S. Suhai, and G. Seifert, Phys. Rev. B 58, 7260 (1998).

${ }^{42}$ T. A. Niehaus, D. Heringer, B. Torralva, and T. Frauenheim, Eur. Phys. J. D 35, 467 (2005).

${ }^{43}$ T. Kubař, U. Kleinekathofer, and M. Elstner, J. Phys. Chem. B 113, 13107 (2009).

${ }^{44}$ T. Kubař and M. Elstner, Phys. Chem. Chem. Phys. 15, 5794 (2013). 
${ }^{45}$ T. Kubař and M. Elstner, J. R. Soc., Interface 10, 20130415 (2013).

${ }^{46}$ S. J. Pal, D. Trivedi, A. V. Akimov, B. Aradi, T. Frauenheim, and O. V. Prezhdo, J. Chem. Theory Comput. 12, 1436 (2016).

${ }^{47}$ R. Mitrić, U. Werner, M. Wohlgemuth, G. Seifert, and V. Bonačić-Koutecký, J. Phys. Chem. A 113, 12700 (2009).

${ }^{48}$ T. Kubař and M. Elstner, J. Phys. Chem. B 114, 11221 (2010).

${ }^{49}$ E. Fabiano, T. W. Keal, and W. Thiel, Chem. Phys. 349, 334 (2008).

${ }^{50}$ Z. Lan, E. Fabiano, and W. Thiel, J. Phys. Chem. B 113, 3548 (2009).

${ }^{51}$ T. Nelson, S. Fernandez-Alberti, V. Chernyak, A. E. Roitberg, and S. Tretiak, J. Phys. Chem. B 115, 5402 (2011).

${ }^{52}$ T. Nelson, S. Fernandez-Alberti, A. E. Roitberg, and S. Tretiak, Acc. Chem. Res. 47, 1155 (2014).

${ }^{53}$ F. Sterpone and P. J. Rossky, J. Phys. Chem. B 112, 4983 (2008).

${ }^{54}$ A. Troisi and G. Orlandi, Phys. Rev. Lett. 96, 086601 (2006).

${ }^{55}$ A. Troisi, Adv. Mater. 19, 2000 (2007).

${ }^{56}$ A. Troisi, J. Chem. Phys. 134, 034702 (2011).

${ }^{57}$ L. Wang and D. Beljonne, J. Chem. Phys. 139, 064316 (2013).

${ }^{58}$ R. A. Marcus, J. Chem. Phys. 24, 966 (1956).

${ }^{59}$ R. A. Marcus, Rev. Mod. Phys. 65, 599 (1993).

${ }^{60}$ Q. Wu and T. Van Voorhis, Phys. Rev. A 72, 024502 (2005).

${ }^{61}$ T. Van Voorhis, T. Kowalczyk, B. Kaduk, L.-P. Wang, C.-L. Cheng, and Q. Wu, Annu. Rev. Phys. Chem. 61, 149 (2010).

${ }^{62}$ A. de la Lande and D. R. Salahub, J. Mol. Struct.: THEOCHEM 943, 115 (2010).

${ }^{63} \mathrm{H}$. Oberhofer and J. Blumberger, J. Chem. Phys. 131, 064101 (2009).

${ }^{64}$ H. Oberhofer and J. Blumberger, J. Chem. Phys. 133, 244105 (2010).

${ }^{65}$ F. Gajdos, S. Valner, F. Hoffmann, J. Spencer, M. Breuer, A. Kubas, M. Dupuis, and J. Blumberger, J. Chem. Theory Comput. 10, 4653 (2014).

${ }^{66}$ A. Kubas, F. Hoffmann, A. Heck, H. Oberhofer, M. Elstner, and J. Blumberger, J. Chem. Phys. 140, 104105 (2014).

${ }^{67}$ A. Kubas, F. Gajdos, A. Heck, H. Oberhofer, M. Elstner, and J. Blumberger, Phys. Chem. Chem. Phys. 17, 14342 (2015).

${ }^{68}$ S. Lany and A. Zunger, Phys. Rev. B 80, 085202 (2009).

${ }^{69}$ I. Dabo, A. Ferretti, N. Poilvert, Y. L. Li, N. Marzari, and M. Cococcioni, Phys. Rev. B 82, 11521 (2010).

${ }^{70}$ L. Kronik, T. Stein, S. Refaely-Abramson, and R. Baer, J. Chem. Theory Comput. 8, 1515 (2012).
${ }^{71}$ B. Kaduk, T. Tsuchimochi, and T. V. Voorhis, J. Chem. Phys. 140, 18A503 (2014).

${ }^{72}$ S. P. McGlynn, Introduction to Applied Quantum Chemistry (Holt Rinehart and Winston, New York, 1972).

${ }^{73}$ J. Blumberger and K. McKenna, Phys. Chem. Chem. Phys. 15, 2184 (2013).

${ }^{74} \mathrm{H}$. Oberhofer and J. Blumberger, Angew. Chem., Int. Ed. 49, 3631 (2010).

${ }^{75}$ L. Y. Zhang, R. A. Friesner, and R. B. Murphy, J. Chem. Phys. 107, 450 (1997).

${ }^{76}$ A. Troisi and G. Orlandi, J. Phys. Chem. B 106, 2093 (2002).

${ }^{77}$ D. Tan and Z. Chen, J. Math. Sci. Math. Educ. 7, 1 (2012).

${ }^{78}$ O. V. Prezhdo and P. J. Rossky, J. Chem. Phys. 107, 5863 (1997).

${ }^{79}$ J. Y. Fang and S. Hammes-Schiffer, J. Phys. Chem. A 103, 9399 (1999).

${ }^{80}$ C. Zhu, S. Nangia, A. W. Jasper, and D. G. Truhlar, J. Chem. Phys. 121, 7658 (2004).

${ }^{81}$ C. Zhu, A. W. Jasper, and D. G. Truhlar, J. Chem. Theory Comput. 1, 527 (2005).

${ }^{82}$ A. W. Jasper, S. Nangia, C. Zhu, and D. G. Truhlar, Acc. Chem. Res. 39, 101 (2006).

${ }^{83}$ N. Shenvi, J. E. Subotnik, and W. Yang, J. Chem. Phys. 134, 144102 (2011).

${ }^{84}$ B. R. Landry and J. E. Subotnik, J. Chem. Phys. 135, 191101 (2011).

${ }^{85}$ B. R. Landry and J. E. Subotnik, J. Chem. Phys. 137, 22A513 (2012).

${ }^{86}$ J. C. Phillips, R. Braun, W. Wang, J. Gumbart, E. Tajkhorshid, E. Villa, C. Chipot, R. D. Skeel, L. Kale, and K. Schulten, J. Comput. Chem. 26, 1781 (2005).

${ }^{87}$ D. A. Case, T. Darden, T. Cheatham III, C. Simmerling, J. Wang, R. Duke, R. Luo, M. Crowley, R. C. Walker, W. Zhang et al., AMBER 10, University of California, San Francisco, 2008.

${ }^{88}$ B. S. Brunschwig, J. Logan, M. D. Newton, and N. Sutin, J. Am. Chem. Soc. 102, 5798 (1980).

${ }^{89}$ M. D. Newton and N. Sutin, Annu. Rev. Phys. Chem. 35, 437 (1984).

${ }^{90}$ M. D. Newton, Chem. Rev. 91, 767 (1991).

${ }^{91}$ A. Nitzan, Chemical Dynamics in Condensed Phases (Oxford University Press, 2006).

${ }^{92}$ J. Blumberger, Chem. Rev. 115, 11191 (2015).

${ }^{93}$ T. Nelson, S. Fernandez-Alberti, A. E. Roitberg, and S. Tretiak, Chem. Phys. Lett. 590, 208 (2013). 